\title{
Non-Uniqueness of Einstein's Special Relativity, and the Inconclusiveness of High Energy (Relativistic) Physics
}

\author{
Georg von Brzeski, Vadim von Brzeski \\ Helios Labs, 945 Hoxett St., Gilroy, CA, USA \\ Email: numberjoy@gmail.com
}

How to cite this paper: von Brzeski, G. and von Brzeski, V. (2021) Non-Uniqueness of Einstein's Special Relativity, and the Inconclusiveness of High Energy (Relativistic) Physics. Journal of Modern Physics, 12, 1295-1345.

https://doi.org/10.4236/jmp.2021.129080

Received: May 9, 2021

Accepted: July 18, 2021

Published: July 21, 2021

Copyright $\odot 2021$ by author(s) and Scientific Research Publishing Inc. This work is licensed under the Creative Commons Attribution International License (CC BY 4.0).

http://creativecommons.org/licenses/by/4.0/

\begin{abstract}
In this paper, we present a new form of "special relativity" (BSR), which is isomorphic to Einstein's "special relativity" (ESR). This in turn proves the non-uniqueness of Einstein's "special relativity" and implies the inconclusiveness of so-called "relativistic physics". This work presents new results of principal significance for the foundations of physics and practical results for high energy physics, deep space astrophysics, and cosmology as well. The entire exposition is done within the formalism of the Lorentz $S L(2 C)$ group acting via isometries on real 3-dimensional Lobachevskian (hyperbolic) spaces $L^{3}$ regarded as quotients $S L(2 C) / S U(2)$. We show via direct calculations that both ESR and BSR are parametric maps from Lobachevskian into Euclidean space, namely a gnomonic (central) map in the case of ESR, and a stereographic map in the case of BSR. Such an identification allows us to link these maps to relevant models of Lobachevskian geometry. Thus, we identify ESR as the physical realization of the Beltrami-Klein (non-conformal) model, and BSR as the physical realization of the Poincare (conformal) model of Lobachevskian geometry. Although we focus our discussion on ball models of Lobachevskian geometry, our method is quite general, and for instance, may be applied to the half-space model of Lobachevskian geometry with appropriate "Lorentz group" acting via isometries on (positive) half space, resulting yet in another "special relativity" isomorphic with ESR and BSR. By using the notion of a homotopy of maps, the identification of "special relativities" as maps from Lobachevskian into Euclidean space allows us to justify the existence of an uncountable infinity of hybrid "special relativities" and consequently an uncountable infinity of "relativistic physics" built upon them. This is another new result in physics and it states that so called "relativistic physics" is unique only up to a homotopy. Finally, we show that "paradoxes" of "special relativities" in either ESR or BSR are simply common distortions of
\end{abstract}


maps between non-isometric spaces. The entire exposition is kept at elementary level accessible to majority of students in physics and/or engineering.

\section{Keywords}

Lobachevskian (Hyperbolic) Geometry, Lorentz Group $S L(2 C)$ Action, "Special Relativity", High Energy (Relativistic) Physics, "Paradoxes", Deep Space Astrophysics, Cosmology

\section{Introduction}

The work we present here deals with maps. The meaning of a map used in mathematics and physics is slightly different; however, its essence is the same. There is an opinion that mathematics studies sets (with additional structures) and maps between those sets. This is true also in physics. However, while the maps studied in mathematics are usually between some abstract mathematical structures, in physics, due to its experimental nature, the range of a map is always some subset of the real numbers.

The subsets of the real numbers the physicists are concerned with are called experimental data. A map from a physical space into real numbers is called a direct map which, in scientific practice, is realized as data acquisition and reduction. The process of data acquisition is itself quite complex. For instance, in High Energy Physics, there are situations where some event of interest has to be singled out from billions of similar events.

Data acquired due to a direct map is an "easy" part of a scientist's job. The more difficult part is to interpret this experimental data, and this involves an inverse map. An inverse map deals with our understanding of what actually happens in physical space(s) from which the direct map data come from. The relation between maps in Mathematics and Physics can be presented as follows:

1) Direct map in Mathematics corresponds to data acquisition in Physics.

2) Inverse map in Mathematics corresponds to data interpretation in Physics.

In this paper, we are concerned with two issues:

1) With the non-uniqueness of maps, i.e. when several, alternative, homotopy equivalent maps exist, resulting in alternative non-unique interpretations of physical phenomena.

2) With distortions introduced in the mapping process, which result in apparent deformations of physical entities, which in turn are seen as paradoxes. See for example, Figure 1 below.

We will address the situation when experimental data come with distortions introduced by certain maps. These distortions are independent of informational noise, but they do depend on the particular map with which the experimental data are interpreted. This results in non-unique quantitative interpretations of physical phenomena. 


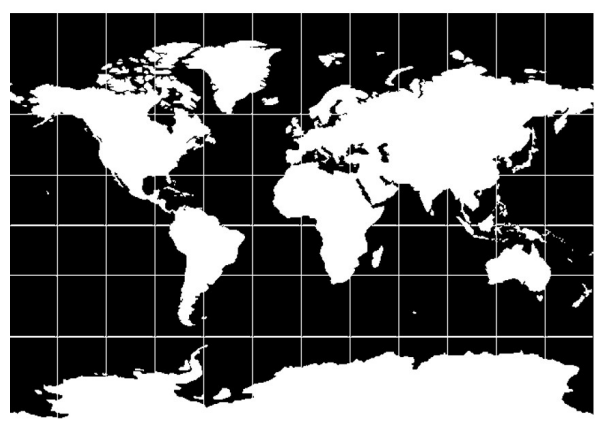

(a)

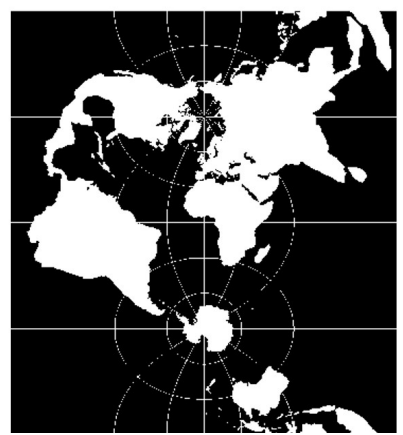

(b)

Figure 1. The figures above show two well known ([Source: Wikipedia Commons]) Mercator maps $S^{2} \rightarrow E^{2}$, standard in (a) and transverse in (b), which are related by homotopy. (The notion of homotopy is discussed in detail in Sections 2.3 and 6). Distortions present in the standard projection are continuously changed into distortions in the transverse projection, as the angle between an axis of the projection cylinder and North-South axis of the sphere $S^{2}$ changes from 0 to 90 degrees. In this paper we discuss analogous mappings and their distortions, however between Lobachevskian and Euclidean spaces, and show how relative apparent sizes of objects due to mapping distortions, particularly in the case of Lobachevskian space mapping, are incorrectly viewed in physics literature as "paradoxes". (a) Standard mercator projection; (b) Transverse mercator projection.

More to the point, we are interested in maps between spaces of the same dimension but with different (constant) curvatures. The non-uniqueness of such interpretations is inherently present in High Energy (high relative velocities) Physics (HEP), and they arise naturally in maps of Lobachevskian negatively curved spaces into a Euclidean flat space.

Lobachevskian (hyperbolic) geometry was developed by several mathematicians, Gauss, Schweikart, Bolay father and son, Beltrami and Lobachevski, just to mention a few. After a two thousand year struggle to prove Euclid's fifth postulate, it appeared around 1835 in closed form due to N.I. Lobachevski, a Russian mathematician of Polish ancestry. A good introduction to Lobachevskian geometry can be found in Anderson [1].

It is needless to say that High Energy Physics (HEP), otherwise known in the literature as "relativistic physics" and "relativistic astrophysics", where relative velocities range from fractions of $c$ to nearly $c$, is based on Einstein's "special relativity" (ESR). Since 1905, it has been generally accepted that phenomena occurring at high relative velocities (with respect to $c$ ) are modeled in unique way (i.e. in the only way possible) by Einstein's "special relativity". Such beliefs resulted in the confidence that the numerical information represented by data gained from ESR indeed reflects the truth about the Nature. This belief, as it will be shown, is misguided.

Since we present a "special relativity" that is mathematically isomorphic but not isometric (or numerically different) from Einstein's "special relativity", in order to avoid confusion, we will label Einstein's "special relativity" as ESR and the authors' "special relativity" as BSR. Both ESR and BSR presented here are 
based on the same group of symmetries-the Lorentz group $S L(2 C)$, acting on its own homogeneous space.

In this paper, we present the following new scientific results, which beyond practical significance, are of utmost importance to the foundation of physics:

1) An alternative to Einstein's "special relativity" (ESR) namely the authors' "special relativity" (BSR) (based on the same Lorentz group).

a) It is shown that BSR corresponds to the Poincare model of Lobachevskian geometry, while Einstein's ESR corresponds to the Beltrami-Klein model of Lobachevskian geometry.

b) It is shown that BSR is equivalent to a stereographic projection from Lobachevskian into Euclidean space, while Einstein's ESR is equivalent to gnomonic (central) projection from Lobachevskian space into Euclidean space.

c) It is shown that both projections, the Poincare (stereographic) and the Beltrami-Klein (gnomonic), result from different actions of the Lorentz group $S L(2 C)$ on its own homogeneous space $S L(2 C) / S U(2)$ isomorphic to a real 3-dimensional Lobachevskian space.

2) Due to above results, we prove the non-uniqueness of Einstein's "special relativity" and the non-uniqueness of any Lorentz group based "relativity" as well.

3) On the basis the homotopy theory, an existence of an uncountable infinity (i.e. continuum) of alternative "special relativities" is proved.

4) On the basis of (3) above, we prove the inconclusiveness of High Energy Physics, or more precisely, the conclusiveness of HEP up to a homotopy only.

5) The so called Twin Paradox is mathematical solved in a symmetric setting showing the apparent distortions introduced by various maps called "special relativities".

6) The problem of images of fast moving circular objects is shown to be a-priori undecidable, being non-unique and dependent on a particular map.

How This Paper Is Organized

Our work is functionally divided into the following sections:

- Section 2: We discuss the properties of maps, specifically of maps between spaces of constant curvature and Euclidean space. This is important because expertise gained from spherical cartography, $K>0$, will be applied to cartography from Lobachevskian space (hyperboloids) $K<0$.

- Section 3: We discuss Lorentz group actions on homogeneous spaces. From the single concept of Lorentz group action, we arrive at our equation of our "special relativity" BSR as well as equations of Einstein's "special relativity" ESR.

- Sections 4 and 5: We show that various "Special Relativities" are simply maps from Lobachevskian Space into Euclidean Space. In particular we study the gnomonic (central) and stereographic maps from spheres and hyperboloids, and establish their relation to Beltrami-Klein model and to Poincare model of Lobachevskian geometry respectively. The isomorphism of the Beltrami-Klein and Poincare models is shown, thus showing the isomorphism between 
Einstein's "Special Relativity" ESR and our "Special Relativity” BSR.

- Section 6: We discuss the homotopy of maps, resulting in a continuously infinite set of possible "special relativities". The existence of infinitely many uncountable "special relativities" is proved via the concept of homotopy.

- Section 7: We discuss several "paradoxes" from relativistic and high energy physics. We show the apparent nature of so-called "relativistic effects" and/or "paradoxes" as distortions resulting from maps between non-isometric spaces of the same real dimension.

A word on notation. We use quite standard notation, however, following Gelfand, Grayev, and Vilenkin, everywhere in this work, we call $S L(2 C)$ the Lorentz group. In older literature, the Lorentz group is $S O(1,3)$, for which $S L(2 C)$ is its double cover.

\section{Maps from Spaces of Constant Curvature into Euclidean Space and Their Properties}

In this section we will give the reader an easy introduction to effects of distortions which are present in maps between non isometric spaces. As we will later see, these distortions in the context of "special relativity" are misunderstood as real phenomena and are represented by variety of "paradoxes".

\subsection{Distortions of Data Due to Maps between Non-Isometric Spaces}

The physics in this work takes place in Lobachevskian negatively curved spaces. Unfortunately we do not perceive negative curvature in the way we perceive the spectral content of a light (colors), or music, or motion around us. Thus there is a need to "translate", to map, to project the results internal to Lobachevskian spaces into the 3-dimensional piece of Euclidean (flat) space of our laboratory. This is a step of utmost importance, since during the mapping we introduce (out of necessity) all kinds of distortions which even today are not properly understood.

Exploration of the world around us, and exploration of the Earth in particular, would be hardly possible without maps. It is essential to understand the different ways or methods of making maps, and the distortions of the images produced by these maps or models.

In ancient times, as long as people did not wander "too far" from their homes, maps drawn on a flat piece of paper were quite faithful. The Earth was believed to be flat just like the piece of a paper. Thus image of the Earth's flat surface on a flat piece of paper was perfect with no distortions of any kind, neither angular nor in relative sizes. Mapping of a flat space onto flat space (of the same dimension) with no distortions is possible because:

Remark 1 All Euclidean spaces of the same dimension are isometric. It follows that maps between Euclidean spaces (of the same dimension) are globally distortion-less on the entire space.

Due to the above property of Euclidean spaces of the same dimension, we 
have in fact only one Euclidean geometry. Therefore, maps between formally different representations of Euclidean geometry via different spaces (of the same dimension) are in fact done "in the same space".

This situation changed when people started to navigate open seas and realized that what they considered as flat was in fact positively curved. Thus for navigational purposes, new map making methods were developed. These methods applied mathematics to get images of the Earth's curved surface on a piece of flat paper, i.e. onto a piece of the Euclidean plane $E^{2}$.

It is important to understand how the distorted image of the Earth's surface we see on a flat map is related to the positively curved surface $S^{2}, K>0$, of the Earth which is a model of the non-Euclidean - spherical geometry in two dimensions. It is important because using examples of positively curved spaces, which intuitively are better perceived than negatively curved ones, we will demonstrate some general mathematical concepts like isometry, isomorphism and conformality. These concepts apply equally well to maps from positively curved spaces and maps from negatively curved spaces. The experience acquired from mapping spaces of constant positive curvature into Euclidean space will make it easy to comprehend mappings of spaces of constant negative curvature into Euclidean space. As we will show, at the base of Einstein's "special relativity" (ESR), our "relativity" (BSR), and all possible other future "relativities" based on the Lorentz group $S L(2 C)$, lies the problem of mapping of non-Euclidean (negatively curved) spaces into Euclidean flat spaces.

In general, when the curvature of the space in question is not constant, the problem of mapping is quite complicated. However, in the case of constant positive curvature, for instance, several ways of mapping have been developed. Many models (images) of the spherical geometry of the Earth's surface in a Euclidean (flat space) exist. These maps, known also as projections, give images of a curved geometry via a flat (Euclidean) geometry. Widely known examples include orthographic, stereographic, gnomonic, and Mercator projections. Each particular map has its own advantages and disadvantages depending on its applications.

Different maps (viewed as sets) from a curved $K=$ const. $\neq 0$ space into a flat $K=0$ space all are isomorphic. However they are not isometric, and some are non-conformal, which means that images resulting from those maps will show distortions in relative sizes and angular relations of mapped objects. As a rule, distortions are larger the larger the chunk of curved space that is mapped into a flat space, so the mapping is faithful only locally. The general rule is:

Remark 2 Maps between spaces of constant nonzero curvature and Euclidean spaces cause distortions, and only locally are they approximately distortion-free. This is because spaces having different constant nonzero curvatures are not isometric, assuming that dimensions of all spaces are equal.

In the case of an $S^{2} \rightarrow E^{2}$ mapping, distortions are determined by the ratio 
$\frac{l}{R}$ of the curved domain's linear size $l$ to the radius $R$ of the ball $B^{3}$ bounded by $S^{2}$. The smaller the ratio $\frac{l}{R} \ll 1$ is (recall the $\frac{v}{c} \ll 1$ regime in ESR), the more faithful the image of that curved surface will be on flat a surface. It is obvious that such maps are neither regarded as "laws of nature" nor as "theories" (as in the "theory of special relativity"), but merely as convenient ways of mapping curved spaces (surfaces) into flat spaces (surfaces).

If we were to regard map making as the business of mathematics, and exploratory journeys due to those maps as physical experiments, then it is obvious that not every map (from all available maps) will be useful for a particular experiment, since some of those maps will produce highly distorted information that is far from reality. For instance, in the case of spherical geometry applied to mapping the Earth's surface, it is quite relevant which map (flat model of the Earth surface) will be used as the tool to navigate the globe. The standard Mercator map is entirely useless to navigate around the poles (e.g. around Greenland or Antarctica) due to excessive distortions (non-linearities); see Figure 1. The Mercator map shows Greenland being as large as Africa, despite Africa actually being 15 times larger than Greenland. Nevertheless, the Mercator map is quite a good model of the Earth's geometry if navigating around low latitudes (i.e. close to the equator).

Methods of mapping the Earth's surface have developed quite well over time. This is because geometric forms of positive curvature are abundant around us, and were known to man since ancient times. Maps of objects of constant positive curvature into Euclidean space were known to artists, map makers, mathematicians for centuries. On the other hand, objects or forms of negative curvature went unknown or unnoticed up to the 19th century, and there were no relevant maps (at least in physics) from spaces of negative curvature into Euclidean spaces. In physics, the first such map was Einstein's ESR, and in art, Escher's paintings. It is no surprise that when ESR appeared in 1905 and was interpreted (unfortunately) via Minkowski's flat geometry-with all its paradoxes-it was quite a shock then and is still a major misunderstanding today.

As in maps from positively curved spaces, we expect that when we map from negatively curved spaces into Euclidean space, we will also experience distortions of images. But since the curvature $K$ is negative this time, the character of distortions will be opposite to those seen in the positive curvature case. Distortions of maps from negatively curved spaces into flat spaces will appear as contractions, instead of expansions.

It is well known in mathematics (see Figure 2) but overlooked by physicists and astronomers, that negatively curved spaces are more volumetric, and positively curved spaces are less volumetric than Euclidean spaces. Specifically:

1) An inhabitant of a Lobachevskian space (a hyperboloid) with $K<0$, who interprets other geometries in terms of his own, will see Euclidean space as contracting and a spherical space as contracting even more. 


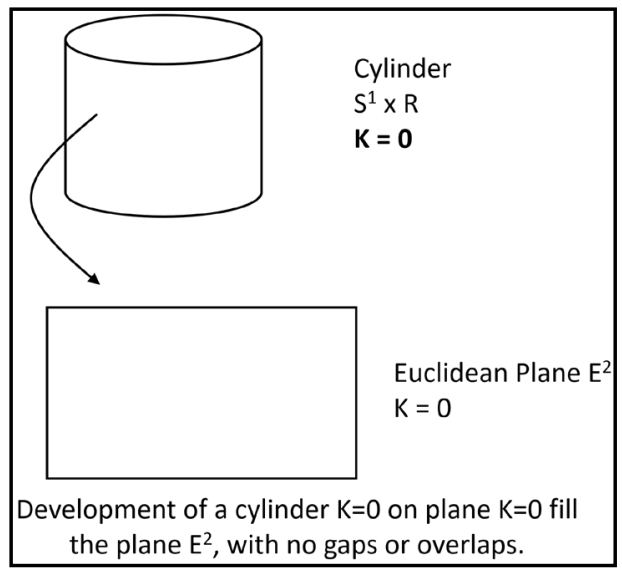

(a)



(b)



(c)

Figure 2. Figures (a)-(c) show the dependence of the volumetric content of spaces of constant Gaussian curvature $K$ versus the sign of their curvatures. We see that spherical space, $K>0$, is less volumetric than Euclidean space $K=0$, while Lobachevskian space $K<0$ is more volumetric than Euclidean space $K=0$. It is interesting to note that this property of Lobachevskian spaces already found an application in data packing techniques and digital signal design (more space - more data) in the Internet domain. 
2) On the other hand, an inhabitant of a spherical universe with $K>0$, who interprets other spaces via the geometry of his own space, will conclude that Euclidean spaces expand and Lobachevskian spaces expand even more.

3) An inhabitant of an Euclidean world with $K=0$, who believes that any other geometry (and physics) has to be interpreted in Euclidean terms, will see a spherical space as Euclidean-contracting, and see the Lobachevskian (hyperbolic) space as Euclidean-expanding.

We live in a world which locally, due to limited resolving power of our instruments, can be regarded as Euclidean. However, experiments clearly show that the physics and geometry of high relative velocities and large distances do not agree with the rules of Euclidean geometry [2] [3] [4]. Since we are dealing with velocities close to $c$ and with distances on cosmological scales, in order to avoid confusion and common misinterpretations, we always need to remember the relation between the sign of the curvature of the spaces and the relative volumes. This is summarized in Table 1 with assumption that the dimension of all entries is the same.

Table 1. The "contraction"/"expansion" effects of images of physical objects or even entire spaces result from comparing incomparable metric relations in non-isometric spaces. The nine possible cases above (read as maps from rows $\rightarrow$ columns) show the apparent "effects" from the lack of consideration of non-Euclidean geometries. For instance, the third-row, second-column entry, "contraction": this is what an observer from a negatively curved space $K<0$ will conclude about a flat space $K=0$. Also, the second-row, third-column entry, "expansion": this is what observer from flat space $K=0$ will conclude about a negatively curved space $K<0$, precisely illustrating the misconception of the so-called "expanding universe" which has been plaguing cosmology for over a hundred years.

\begin{tabular}{|c|c|c|c|}
\hline & $K=+1$ & $K=0$ & $K=-1$ \\
\hline$K=+1$ & isometry & “expansion” & "expansion" \\
\hline$K=0$ & "contraction" & isometry & "expansion" \\
\hline$K=-1$ & "contraction" & "contraction" & isometry \\
\hline
\end{tabular}

\subsection{Non-Uniqueness of Data Interpretation Due to Maps from Spaces of Constant Curvature into Euclidean Space}

The aim of this section is to show how a continuous and uncountable infinite family of parametric maps, dependent on a real parameter, arise from maps of spaces of constant curvature into Euclidean space. The real parameter a on which the map depends produces a gnomonic (central) map and a stereographic map at its two particular extreme values. All other values of the parameter in between the extremes correspond to an uncountable infinity of a mixed map. The uncountable infinity of possible maps is expressed in a natural way by the notion of homotopy [3] which, in an informal way, is introduced in Subsection 2.3. 


\subsubsection{Spherical Cartography}

To get more insight into cartography from a sphere via different projections, it will be natural to use the language of projective geometry. (Projective geometry was developed in the 19-th century for the needs of pure geometry; today it is the basic tool for computer graphics.)

Regarding our discussion, projective geometry encompasses both cases of spherical and Lobachevskian (hyperbolic) geometry. It gives an elegant and uniform way to treat spherical and Lobachevskian cartography by appropriate (to each case) normalization of projective coordinates. We present the spherical cartography case below, whereas the Lobachevskian cartography case is discussed in 2.2.2.

For the sake of simplicity only, we will consider the one dimensional case, namely the real projective line $R P^{1}$, and its mapping into an affine line $E^{1}$.

On the projective line $R P^{1}$ we have to deal with projective coordinates. Since in a real projective space of dimension $n$ there are $n+1$ projective (homogeneous) coordinates, it follows that on a projective line which has dimension one, there will be two projective coordinates which we denote as $\xi_{0}$ and $\xi_{3}$. Subscripts are irrelevant but later on we will see why they are chosen in this way. Of course in any space of dimension $n$, there can only be $n$ linearly independent coordinates. That redundancy, in the case of projective coordinates, is handled by imposing some kind of normalization condition which we choose as:

$$
\xi_{0}^{2}+\xi_{3}^{2}=1
$$

Normalization condition (1) results in only one independent coordinate and (in projective coordinates) is the equation of the unit circle centered at $o(0,0)$.

The single (local) coordinate on an affine line into which the projection is done is denoted by $x$. Affine $x$ and projective coordinates $\xi$ are related as:

$$
x=\frac{\xi_{3}}{\xi_{0}-a}
$$

The parameter $a$ in (2) has a simple meaning. From the point of view of geometry, it is equal to the Euclidean distance $\mathbf{d}(\mathbf{o}, \mathbf{p})$ between the center of the unit sphere $o$ and the center of projection $p$, so $a=d(o, p)$. The parameter a determines the kind of projection or a type of a map. Since in the present work we are interested in gnomonic and stereographic projections (and hybrid projections as well) the parameter a will be limited to values listed below. From the point of view of topology, parameter $a$ is a variable homotopy parameter which determines the continuous deformation of one map into another [5]. As a changes continuously through its range, the maps change (deform) accordingly, something known in mathematics as the homotopy of maps, which we will discuss in more detail later on.

In this paper we concern ourselves with the following cases of the parameter $a$ : - $a=0 \Rightarrow$ gnomonic (central) projection.

- $a \in(0,1) \Rightarrow$ continuum of hybrid (mixed) projections, mixed maps.

- $a=1 \Rightarrow$ stereographic projection, stereographic map. 
$a=0:$ The case of gnomonic (central) projection, from a projective line into an affine line.

We can express projective coordinates via coordinate(s) $x$ by solving Equations (1) and (2) with $a=0$. This gives:

$$
\xi_{0}=\frac{1}{\sqrt{1+x^{2}}}, \quad \xi_{3}=\frac{x}{\sqrt{1+x^{2}}}
$$

In general, in an $n$-dimensional case of a gnomonic projection from an $n$-dimensional projective space into an $n$-dimensional affine space:

$$
\xi_{0}=\frac{1}{\sqrt{1+|x|^{2}}}, \quad \xi_{i}=\frac{x_{i}}{\sqrt{1+|x|^{2}}}, \quad i=1, \cdots, n
$$

We call projective (homogeneous) coordinates is the form (4) Weierstrass coordinates since they are analogous to those used by Weierstrass in his work on Lobachevskian (hyperbolic) geometry [6] which we will explicitly derive in the next section.

$a=1$. The case of stereographic projection, from a projective line into an affine line.

Solving Equations (1) and (2) with $a=1$, we find the projective coordinates expressed via affine coordinate(s) due to stereographic map from a projective to an affine line. Those are:

$$
\xi_{0}=\frac{1-x^{2}}{1+x^{2}}, \quad \xi_{3}=\frac{2 x}{1+x^{2}}
$$

In general, in an $n$-dimensional case of stereographic projection from an $n$-dimensional projective space into an $n$-dimensional affine space:

$$
\xi_{0}=\frac{1-|x|^{2}}{1+|x|^{2}}, \quad \xi_{i}=\frac{2 x_{i}}{1+|x|^{2}}, \quad i=1, \cdots, n
$$

In both cases, in angular coordinates:

$$
\xi_{0}=\cos \alpha, \quad \xi_{3}=\sin \alpha
$$

Projective coordinates in a form of (6) are called rational coordinates and are related to a stereographic projection. They are also referred to as the rational parametrization of a circle. It is obvious that in all three cases (3), (5), and (7), $\xi_{1}^{2}+\xi_{3}^{2}=1$.

In Figure 3 and Figure 4, the gnomonic and stereographic projections from the unit radius sphere into Euclidean space are shown. The aim of Figure 3 and Figure 4 is to show that the data exchange between a spherical space $K>0$ and a Euclidean space $K=0$ cannot be interpreted in unique way. Moreover, we see that the data due to a gnomonic projection are $D_{g}=\tan \alpha$, while data due to stereographic projection are $D_{s}=2 \tan \frac{\alpha}{2}$. Since:

$$
\tan \alpha=\frac{2 \tan \frac{\alpha}{2}}{1-\tan ^{2} \frac{\alpha}{2}}
$$




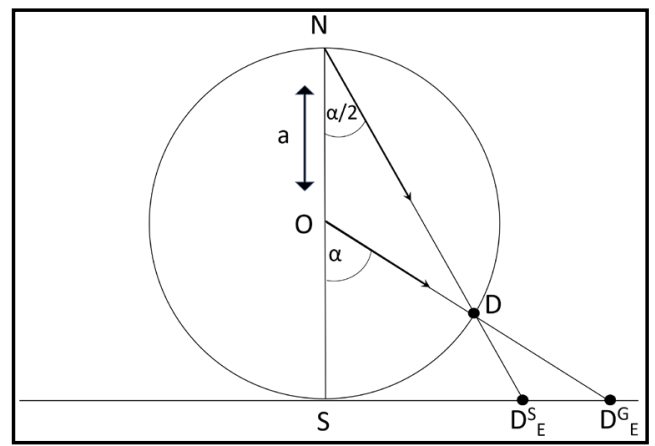

Figure 3. Spherical Space Mapping, $S^{2}(K>0) \rightarrow E^{2}(K=0)$. The single data $D$ from spherical space are mapped onto two different data $D_{E}^{S}$ and $D_{E}^{G}$ in Euclidean space of a physicist's laboratory. Data $D_{E}^{S}$ are due to a stereographic map while data $D_{E}^{G}$ are due to a gnomonic map. Between $D_{E}^{S}$ and $D_{E}^{G}$ (one the segment $\left(D_{E}^{S}, D_{E}^{G}\right)$ ) there is a continuum of Euclidean data corresponding to hybrid maps when the projection point takes an arbitrary position on the segment $O N$.

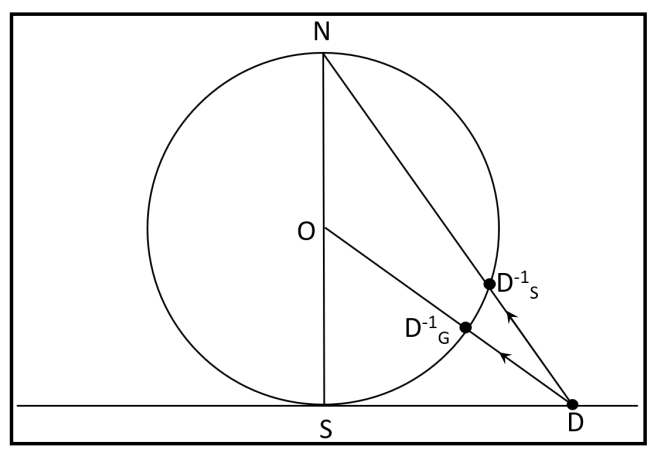

Figure 4. Spherical Space Mapping, $E^{2}(K=0) \rightarrow S^{2}(K>0)$, the inverse map. The single data $D$ in the Euclidean space of a physicist's laboratory is mapped onto two different data $D_{G}^{-1}$ and $D_{S}^{-1}$ in spherical space. Data $D_{S}^{-1}$ are due to a (inverse) stereographic map, while data $D_{G}^{-1}$ result from a (inverse) gnomonic map. Between $D_{S}^{-1}$ and $D_{G}^{-1}$ on the segment $\left[D_{S}^{-1}, D_{G}^{-1}\right]$ there is a continuum of spherical space data corresponding to a single Euclidean datum due to (inverse) hybrid maps. This illustrates the state of the undecidable situation when physicists are unable to decide which data in spherical space correspond (in a unique way) to the data they acquired in Euclidean space. In the language of cause and effect, it is impossible to single out a particular cause which results in a detected effect. This indeterminate situation is typical of quantum mechanics. We return to this below when we discuss "relativities" as maps.

or:

$$
D_{g}=\frac{D_{s}}{1-\left(\begin{array}{c}
D_{s} \\
2
\end{array}\right)^{2}}
$$

The inverse map is given as:

$$
\tan \frac{\theta}{2}=\frac{\tan \theta}{1+\sqrt{1-\tan ^{2} \theta}}=\frac{\left(1-\sqrt{1-\tan ^{2} \theta}\right)}{\tan \theta}
$$


Equations (8) and (10) give an isomorphism between a gnomonic map and a stereographic map of a unit sphere onto the Euclidean plane.

$$
\text { Gnomonic } \Leftrightarrow \tan \theta \overleftrightarrow{I S O} \tan \frac{\theta}{2} \Leftrightarrow \text { Stereographic }
$$

These facts will appear again when we discuss "special relativities" as gnomonic and stereographic maps.

Conclusion 3 From the formulas of two alternative parametrizations of the unit circle (3) and (5) and from Figure 3 and Figure 4, we note that the parametrization of a circle (a 1-dimensional spherical space) by a full parameter $\alpha$ corresponds to a gnomonic (central) map, while a parametrization of a unit circle by a half parameter $\alpha / 2$ corresponds to a stereographic map.

At the end of this section we would like the reader to remember the following:

1) A central or gnomonic projection (map) from a sphere, $K>0$, is related to the Weierstrass parametrization of a unit sphere.

2) A stereographic projection from the a sphere, $K>0$, is related to the rational parametrization of the unit sphere.

\subsubsection{Lobachevskian (Hyperbolic) Cartography}

We now repeat the derivation from Section 2.2.1 but this time for Lobachevskian space; see Figure 5. Again, consider the projective line $R P^{1}$ equipped with projective coordinates $u_{0}, u_{3}$ subject to normalization condition (12):

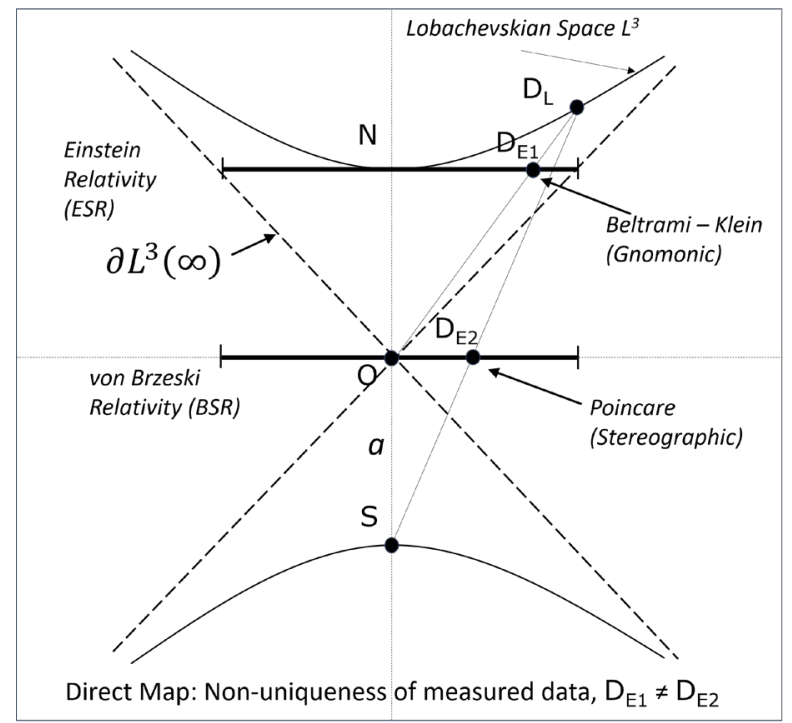

Figure 5. Lobachevskian (hyperbolic) Space Mapping, $L^{2}(K<0) \rightarrow E^{2}(K=0)$. The same single data $D_{L}$ in Lobachevskian space are mapped onto two different data $D_{E 1}$ and $D_{E 2}$ into Euclidean space of physicist laboratory. Data $D_{E 1}$ are due to gnomonic map while data $D_{E 2}$ are due to stereographic map. Between $D_{E 1}$ and $D_{E 2}$ there is a continuum of Euclidean data corresponding to hybrid maps when projection point takes arbitrary position on $[-1,0]$ segment. Data $D_{E 1}$ and $D_{E 2}$ are related by isomorphism as $D_{E 1}=\frac{2 D_{E 2}}{1+D_{E 2}^{2}}$. 


$$
u_{0}^{2}-u_{3}^{2}=1
$$

which in projective coordinates $u_{0}$ and $u_{3}$ is a two-branched unit hyperbola centered $O(0,0)$.

In order to be closer to what will follow, we take the Lobachevskian line as a 1-dimensional Lobachevskian velocity space, in which points represent velocities and distances between points represent relative velocities. This time the affine coordinate (depending on the projection from a Lobachevskian line to an affine line) will be denoted as $\mathrm{v}$ for a gnomonic (central) projection and as $v$ for a stereographic projection. The affine (local) coordinates $\mathbf{v}$ and $v$ are the measurable data which relativistic physics acquires from experiments. The parameter a represents the distance between the center of the hyperbola and the center of projection, and takes values $a=0,0<a<1, a=-1$, yielding:

- $a=0 \Rightarrow$ a gnomonic projection.

- $a \in(-1,0) \Rightarrow$ a continuum of hybrid projections (mixed maps).

- $a=-1 \Rightarrow$ a stereographic projection.

Depending on the parameter $a$, the affine coordinates $\mathrm{v}$ and $v$ are expressed, via projective coordinates, as:

$$
\mathrm{v} \text { or } v=\frac{u_{3}}{u_{0}-a}
$$

$\mathrm{v}$ in the case of $a=0$, and $v$ in the case of $a=-1$, respectively; see Figure 5.

The reader is encouraged to repeat the calculations from previous section to see that:

$\mathrm{a}=0$. The case of gnomonic projection, from a Lobachevskian line into an affine line. Solutions of Equations (12) and (13) give:

$$
u_{0}=\frac{1}{\sqrt{1-\mathrm{v}^{2}}}, \quad u_{3}=\frac{\mathrm{v}_{3}}{\sqrt{1-\mathrm{v}^{2}}}|\mathrm{v}| \in(0,1)
$$

and in the general $n$-dimensional case of a gnomonic (central) projection from an $n$-dimensional Lobachevskian space into an $n$-dimensional affine space:

$$
u_{0}=\frac{1}{\sqrt{1-|\mathrm{v}|^{2}}}, \quad u_{i}=\frac{\mathrm{v}_{i}}{\sqrt{1-|\mathrm{v}|^{2}}}, \quad i=1, \cdots, n, \quad|\mathrm{v}| \in(0,1)
$$

Projective coordinates in the form (15) are known as Weierstrass coordinates as mentioned earlier, which Weierstrass developed and used at least 50 years before Einstein's ESR. An alternative parametrization via hyperbolic functions yields:

$$
u_{0}=\cosh \theta, \quad u_{3}=\sinh \theta
$$

It is easy to check that both (14) and (16) obey the normalization condition (12). From (13) we see that the affine coordinate $\mathrm{v}$ at $a=0$ is equal to:

$$
\frac{u_{3}}{u_{0}}=\mathrm{v}=\tanh \theta
$$

where $\theta$ is the (signed) distance in the Lobachevskian line, meaning Loba- 
chevskian relative velocity. Note that while $0<|\theta|<\infty, 0<v<1$. Values $\theta=\infty$ and $v=1$ belong to the boundary at infinity of a Lobachevskian line and are interpreted as velocities of photons.

$a=-1$. The case of stereographic projection, from a Lobachevskian line into an affine line. Similarly to the previous paragraph, we obtain:

$$
u_{0}=\frac{1+v^{2}}{1-v^{2}}=\cosh \theta, \quad u_{3}=\frac{2 v}{1-v^{2}}=\sinh \theta
$$

Coordinates (18) are rational projective coordinates, which give the rational parametrization of a unit hyperbola and are analogous to the rational parametrization of a unit circle (5). Rational projective coordinates in the Lobachevskian case are related to stereographic projection from a hyperboloid as rational coordinates in the spherical case are related to a stereographic projection from a sphere.

The generalization of Formula (18) in an $n$-dimensional Lobachevskian space is:

$$
u_{0}=\frac{1+|v|^{2}}{1-|v|^{2}}, \quad u_{i}=\frac{2 v_{i}}{1-|v|^{2}}, \quad i=1, \cdots, n, \quad|\mathrm{v}| \in(0,1)
$$

Next, from (17) and (18) we see that:

$$
\frac{u_{3}}{u_{0}}=\tanh \theta=\mathrm{v}=\frac{2 v}{1+v^{2}}
$$

The formula

$$
\mathrm{v}=\frac{2 v}{1+v^{2}}
$$

shows a well known relation of isomorphism between the Beltrami-Klein and Poincare models of Lobachevskian geometry, which is represented here by Lobachevskian velocity space.

Next, since:

$$
\tanh \frac{\theta}{2}=\left(\frac{\cosh \theta-1}{\cosh \theta+1}\right)^{1 / 2}
$$

from (22), we find the Euclidean velocity $v$ versus Lobachevskian velocity $\theta$, in the case of a stereographic map, is:

$$
v=\tanh \frac{\theta}{2}
$$

Thus the relations of isomorphism between a gnomonic and a stereographic projection are:

1) In terms of Lobachevskian velocity space:

$$
\tanh \theta=\frac{2 \tanh \frac{\theta}{2}}{1+\tanh ^{2} \frac{\theta}{2}}
$$




$$
\tanh \frac{\theta}{2}=\frac{\tanh \theta}{1+\sqrt{1-\tanh ^{2} \theta}}=\frac{1-\sqrt{1-\tanh ^{2} \theta}}{\tanh \theta}
$$

See the analogy with spherical cartography.

2) In terms of Euclidean velocity space (that the physicist deals with):

$$
\begin{gathered}
\mathrm{v}=\frac{2 v}{1+v^{2}} \\
v=\frac{\mathrm{v}}{1+\sqrt{1-\mathrm{v}^{2}}}=\frac{1-\sqrt{1-\mathrm{v}^{2}}}{\mathrm{v}} \\
\text { Beltrami-Klein } \ni \tanh \theta \overleftrightarrow{I S O} \tanh \frac{\theta}{2} \in \text { Poincare }
\end{gathered}
$$

The arrow over ISO indicates both ways of mapping due to the isomorphism between gnomonic and stereographic images from the (unit) hyperboloid into the plane $E^{2}$.

The above simple calculations have led to a very important and well known conclusion:

1) The Beltrami-Klein model corresponds to a gnomonic (central) projection from Lobachevskian into Euclidean space.

2) The Poincare model corresponds to a stereographic projection from Lobachevskian to Euclidean space.

So far, performing the calculations in spherical and Lobachevskian cartographies, we have not mentioned anything at all about the Lorentz group or Lorentz transformations. In the following exposition (Section 3), we will obtain the same results via Lorentz group action (Lorentz transformations) on Lobachevskian space, resulting in "Special Relativities" which will be identified as maps (gnomonic, stereographic, or mixed).

At the end of this section we would like the reader to remember that:

1) The central or gnomonic projection (map) from Lobachevskian spaces (hyperboloids), $K<0$, into Euclidean space is related to the Weierstrass parametrization of the unit hyperboloid.

2) The stereographic projection (map) from Lobachevskian spaces (hyperboloids), $K<0$, into Euclidean space is related to the rational parametrization of a unit hyperboloid.

A nice discussion of hyperbolic cartography can be found in Reynolds [7].

\subsection{Homotopy and an Infinity of Maps}

A homotopy is a topological notion. It is quite important and useful for our exposition. It is analogous to the notion of homeomorphism, but it is coarser than homeomorphism. A formal definition will be given in Section 6. While a homeomorphism relates topological spaces, a homotopy relates continuous maps, which can be regarded as "points" in a space of continuous mappings. In terms of equivalence, a homotopy is an equivalence relation on a space of continuous mappings, similarly like homeomorphism is an equivalence relation on topolog- 
ical spaces.

Mappings, or projections in general, depend on the position of the projection point, also known as the center of projection. Since we can continuously change the projection point, it follows that all such maps, viewed as a points in a so-called mapping space are uncountably infinite and can be changed one into another one continuously. In other words, they are all homotopic. The reader should note that these topological properties of maps apply to maps from spheres as well as from hyperboloids (Lobachevskian spaces).

We believe it is worth looking at the general picture via a homotopy of maps, which we will later apply to maps from Lobachevskian space $K<0$ into Euclidean space $K=0$. A homotopy of maps, simply put, is the continuous deformation of one continuous map into another continuous map. Spaces due to homotopic maps are of the same homotopy type [3], which roughly means that one space can be continuously deformed onto another.

Consider the gnomonic and stereographic projections in case of the sphere $S^{2}$ into the Euclidean plane $E^{2}$. The point from which the projection is done in a gnomonic map is the center of a sphere $S^{2}$, while in a stereographic map the projection is done from "the North Pole" point $N$, Figure 3. If we start with a gnomonic map and move the point of projection continuously up from the center of the sphere along the radius toward the North Pole $N$ we will have a continuous family of hybrid maps. When the point of projection reaches to the North Pole $N$ we will have a stereographic projection. You may imagine the whole process as a continuous deformation of the gnomonic map into stereographic map. We see that points $p$ along the unit segment continuously parametrize maps of $S^{2}$ into $E^{2}$. Since on the unit segment there is an continuum of points (uncountable infinity), there is therefore a continuum of maps of a spherical space, $K>0$, into the Euclidean space $E^{2}$. In regards to our exposition, we can say that images of the Earth's surface $S^{2}$ due to gnomonic and stereographic maps are of the same homotopy type, i.e. images of $S^{2}$ in $E^{2}$ are homotopy equivalent.

Regarding the distortions of maps from curved into flat spaces, we recall that:

1) Maps from curved spaces into Euclidean space are not unique and not isometric. They will show distortions depending on the curvature of the initial space, on the method of mapping, and on the linear size of the mapped domain.

2) Maps (images) from positively curved spaces, $K>0$, e.g. from the Earth's surface, into the Euclidean plane $K=0$ will show images of distortions of enlargement, or images of expansion of objects, since a space of zero curvature (flat) is more volumetric than a positively curved space.

3) Conversely, maps from a Euclidean space into spherical space will show distortions as compression, and an contraction of images.

4) Maps (images) from Lobachevskian negatively curved spaces (hyperboloids), $K<0$, into an Euclidean space, $K=0$, will show distortions of compression, or an contraction of images of objects ("Fitzgerald contraction", "time dilation"). This is because Euclidean space is less volumetric than a hyperbolic 
negatively curved (Lobachevskian) space.

5) Conversely, maps from an Euclidean space into a Lobachevskian space will show distortions of enlargement, or distortions of expansion.

\section{Lobachevskian Homogeneous Spaces Related to the Lorentz Group and Lorentz Group Action}

This section contains some already known material [8] [9] [10]. It is included as a convenience for the reader only.

Definition $4 A$ space $X$ with a given group acting on $X$ is called a homogeneous space if any two points $x, y \in X$ can be joined by some $g \in G$, meaning $y=g x$ for any $x, y \in X$ and some $g \in G$. The group $G$ is called the group of motions of the space $X$.

Homogeneous spaces are very useful in mathematics and physics. Due to ideas going back to the German geometer Feliks Klein [11], homogeneous space $X$ can be described solely in terms of the symmetry group $G$ acting on it. Due to this construction, a homogeneous space inherits many useful properties which belong to a group associated with it.

The procedure itself is as follows. Take some arbitrary point $o \in X$ (call it the origin) and find a subgroup $H \subset G$ which leaves point $o$ unchanged, $H o=o$. That subgroup $H$ is called a stabilizer of the point $o$. The choice of a point $o$ is irrelevant. If one thinks, for instance, about a homogeneous space as of a sphere $S^{2}$ then any point on the sphere may be regarded as the "origin".

After the stabilizer has been found, the quotient space $G / H$ is constructed in which "points" are identified with copies of $H$ shifted by elements of $G$, namely $x_{0}=e H, x_{1}=g_{1} H, \quad x_{2}=g_{2} H, \cdots$ This one to one correspondence between points $x_{i}$ and cosets $g_{i} H$ establishes an isomorphism between the spaces $X$ and $G / H$.

We are interested in a real Lobachevskian 3-dimensional space(s) and a group of motions associated with it which is the Lorentz group $S L(2 C)$. First, a few definitions:

Definition 5 The Lorentz group $S L(2 C)$ is the group of $2 \times 2$ complex

matrices $g=\left(\begin{array}{ll}\alpha & \beta \\ \gamma & \delta\end{array}\right), \alpha, \beta, \gamma, \delta \in C$, with unit determinant $\operatorname{det} g=1$. Since $\operatorname{det} g=1$, as a topological space, $S L(2 C)$ may be viewed as a hyperboloid which is a generic representation of Lobachevskian space. $S L(2 C)$ is a double cover of $S O(1,3)$ which is used in orthodox "special relativity" in Minkowski pseudo-Euclidean real 4-dimensional flat geometry.

Definition 6 Lobachevskian (real) space is a simply connected, non-compact, locally compact, metric space of constant Gaussian curvature $K<0$. The generic value of $K$ is usually set to $-1, K=-1$.

Note that compact negatively curved spaces are not Lobachevskian spaces in the sense of Definition 6 and are not discussed here.

We will use two parallel representations of Lobachevskian space: 
1) As an upper sheet of a $3 d$-hyperboloid equipped with projective (homogeneous) coordinates

$$
\xi_{i}, i=0,1,2,3, \xi_{0}^{2}-\xi_{1}^{2}-\xi_{2}^{2}-\xi_{3}^{2}=1, \quad \xi_{0} \geq 1 .
$$

2) As a set $H^{+}$of positive definite Hermitian matrices in $S L(2 C)$, $H^{+} \subset S L(2 C)$. Positive definite matrices are those which have all eigenvalues positive.

In the second representation above, points in the real 3-dimensional Lobachevskian space are identified with complex $2 \times 2$ positive definite Hermitian matrices (with unit determinant), $\tilde{\xi}=\left(\begin{array}{ll}\xi_{0}-\xi_{3} & \xi_{2}-i \xi_{1} \\ \xi_{2}+i \xi_{1} & \xi_{0}+\xi_{3}\end{array}\right)$, where $\xi$ $\left(\xi_{0}, \xi_{1}, \xi_{2}, \xi_{3}\right)$ are projective (homogeneous) coordinates in Lobachevskian real $3 d$-space normalized as $\operatorname{det} \tilde{\xi}=1=\xi_{0}^{2}-\xi_{1}^{2}-\xi_{2}^{2}-\xi_{3}^{2}$.

A one-to-one (bijective) correspondence between projective coordinates $\xi$ and matrices in $H^{+}$is given as:

$$
\tilde{\xi}=\left(\begin{array}{cc}
\xi_{0}-\xi_{3} & \xi_{1}-i \xi_{2} \\
\xi_{1}+i \xi_{2} & \xi_{0}+\xi_{3}
\end{array}\right)=\xi_{\alpha} \sigma^{\alpha}
$$

and

$$
\xi_{\alpha}=\frac{1}{2} \operatorname{Tr}\left(\tilde{\xi} \sigma^{\alpha}\right)
$$

where $\sigma^{0}=e$ is the identity $2 \times 2$ matrix and $\sigma^{k}, k=1,2,3$ are Pauli matrices, and summation is over the same indexes.

Remark 7 The advantage of working with $2 \times 2$ positive definite Hermitian matrices $\tilde{\xi}, \xi=u$ or $\xi=x$ of unit determinant is that: first, matrices $\tilde{u}$ and $\tilde{x}$ represent points $u$ and $x$ in real 3-dim Lobachevskian space $L_{U}^{3}$ and $L_{X}^{3} \quad$ respectively. Projective (homogeneous) coordinates of points $u$ or $x$ are given explicitly via Formula (30). Matrices $\tilde{u}$ and $\tilde{x}$ are also viewed as Hermitian operators acting via isometries (Lorentz group, $S L(2 C)$ actions) on a real 3-dim Lobachevskian space. We do not introduce any special notation to distinguish between the above two cases since the meaning of $\tilde{u}$ and $\tilde{x}$ is clear from the context.

Before we show how to find a stabilizer for the Lobachevskian space $H^{+} \subset S L(2 C)$ we need to define the group actions, called transformations in the physics literature. We describe two types of actions we use in this work.

1) The left action, also called a left translation of the Lorentz group on Lobachevskian space $L_{X}^{3} \quad[10][12]$.

$$
h=g h^{\prime}, \quad h, h^{\prime} \in L_{X}^{3}, \quad g \in S L(2 C)
$$

2) The double sided action, or two sided translation, (sometimes called a similarity transformation) on Lobachevskian space $L_{X}^{3} \quad[10]$.

$$
h=g^{*} h^{\prime} g, \quad h, h^{\prime} \in L_{X}^{3}, g \in S L(2 C)
$$

The star superscript denotes Hermitian conjugation $g \rightarrow g *=\bar{g}^{T}$. 
Remark 8 In the case of double sided action (32), we see that matrices $g$ and $-g$ result in the same motion and have to be identified. This is done by taking the quotient $S L(2 C) / Z(e,-e)=P S L(2 C)$, resulting in the projective Lorentz group PSL $(2 C)$. The center (of the $S L(2 C)$ ) $Z$ is also called the kernel of non-effectiveness. Since $Z$ is discrete, $S L(2 C) \rightarrow P S L(2 C)$ is a covering map. Note that PSL $(2 C)$ is isomorphic with the (proper) Lorentz group $S O(1,3)$ acting on a flat pseudo-Euclidean space. Keeping in mind that two matrices in $S L(2 C)$ which differ only by a sign induce the same Lorentz transformation, we still will use $S L(2 C)$ notation instead of PSL(2C) for this case.

Definition 9 Motion due to action $g \in S L(2 C)$ on a real 3-dim Lobachevskian space $L_{\xi}$ given either by Formula (31) or Formula (32), sends a point $\xi^{\prime} \in L_{\xi}^{3}$ having homogeneous coordinates $\xi_{0}^{\prime}, \xi_{1}^{\prime}, \xi_{2}^{\prime}, \xi_{3}^{\prime}$ represented by matrix $\tilde{\xi}^{\prime}$ onto a point $\xi \in L_{\xi}^{3}$ having homogeneous (projective) coordinates $\xi_{0}, \xi_{1}, \xi_{2}, \xi_{3}$ represented by matrix $\tilde{\xi}$.

Now it is easy to find a stabilizer. Take a unit matrix $e \in H^{+}$as the "center" of Lobachevskian space, $e=\left(\begin{array}{ll}1 & 0 \\ 0 & 1\end{array}\right)$ and apply the Lorentz group motion (Lorentz transformation) to it. From the definition of a stabilizer, we have:

$$
e=g^{*} e g=g^{*} g
$$

which says that matrix $g$ is unitary. Thus we have the following conclusion:

Conclusion 10 The stabilizer of the "origin" $e$ in Lobachevskian 3-dim real space is a group of unitary $2 \times 2$ matrices $S U(2)$, or any conjugates to it in $S L(2 C)$ if another "origin" than e has been selected.

Thus we arrive at another definition of Lobachevskian space:

Definition 11 Real 3-dim Lobachevskian space $H^{+}$is isomorphic with the coset space of the Lorentz group $S L(2 C)$ with respect to $S U(2)$. The group $S L(2 C)$ is the group of rigid motions (isometries) of $\mathrm{H}^{+}$.

$$
S L(2 C) \supset H^{+} \simeq S L(2 C) / S U(2)
$$

where $\simeq$ denotes isomorphism which we will later, with some abuse of notation, denote with an equal sign.

In this representation, Lobachevskian space can be viewed as covered by (collapsed to a point) copies of $S U(2)$ and translated over the entire Lobachevskian space $H^{+}$by the action of a group $S L(2 C)$. Note that $S U(2)$ is locally homeomorphic to the rotation group $S O(3)$.

Further in this paper we use two physical representations of Lobachevskian real 3-dim space, either expressed in projective coordinates or matrix coordinates. We call one the coordinate or position Lobachevskian space, which we denote as $L_{X}^{3}$, and the other one the Lobachevskian velocity/momentum space, which we denotes as $L_{U}^{3}$. The geometry of both is identical, and with respect to EM fields, both representations will cause the same effects. For instance, the redshift in EM spectra may result either from $L_{X}^{3} \quad$ (in which case it is called cosmological redshift), and/or from $L_{U}^{3}$ (in which case it is called the 
Doppler shift), or from both at the same time; see also Section 4.2.4. Therefore, we discuss these two representations of Lobachevskian space in more detail 3.1 and 3.2 below.

At the end of this section, the reader is encouraged to remember that:

1) The Lorentz group is the group $S L(2 C)$.

2) Lobachevskian (hyperbolic) real 3-dim space is a coset space of Lorentz $S L(2 C)$ group with respect to $S U(2) . L^{3}=S L(2 C) / S U(2)$.

3) The Lorentz group acts on its own homogeneous space $S L(2 C) / S U(2)$ via isometries (31) and (32), also called rigid motions.

4) In physics, the action of a Lorentz group is called a Lorentz transformation.

\subsection{Representation of Lobachevskian Geometry as a 3-Dim}

$$
\text { Position Space } L_{X}^{3} \sim S L(2 C) / S U(2)
$$

In the case of Lobachevskian position space $L_{X}^{3}, \xi=x$, we will represent points in 3-dim Lobachevskian position space either via projective (homogeneous) coordinates $x, x_{0}^{2}-x_{1}^{2}-x_{2}^{2}-x_{3}^{2}=1, x_{0} \geq 1$, or via $2 \times 2$ positive definite Hermitian matrices $\tilde{x}$, with a unit determinant, assembled from homogeneous coordinates.

$$
\tilde{x}=\left(\begin{array}{ll}
x_{0}-x_{3} & x_{1}-i x_{2} \\
x_{1}+i x_{2} & x_{0}+x_{3}
\end{array}\right), \quad \operatorname{det} \tilde{x}=1, \quad x_{0} \geq 1
$$

There are several equivalent definitions of positive definite Hermitian matrices. We adopt the one in which a positive definite (Hermitian) matrix is a one with all positive eigenvalues. Thus, we have the following isomorphism between real Lobachevskian 3-dim space $L^{3}$, the set of positive definite Hermitian matrices $H \subset S l(2 C)$, and the quotient space $S L(2 C) / S U(2)$ [10]:

$$
L_{X}^{3}=S L(2 C) / S U(2)
$$

Equation $\operatorname{det} \tilde{x}=x^{2}=1, \quad x_{0} \geq 1$ is the equation of the upper sheet of a two sheet hyperboloid, which in turn is the generic model of a real 3-dim Lobachevskian space. It follows that transformations executed by the Lorentz group, due to conditions $x^{2}=$ invariant , are restricted to the Lobachevskian space $x^{2}=$ constant $>0 \quad$ (constant is usually set to unity-a common choice in curvature normalization).

In a projective representation (Beltrami - Klein ball model), Lobachevskian space is realized on the hyperplane $x_{0}=1$; see Figure 5 . The hyperplane $x_{0}=1$ intersects the cone $x^{2}=0$, along the Euclidean sphere $S^{2}(0, r)$ which represents the boundary at infinity $\partial L_{X}^{3}(\infty)$, for $L_{X}^{3}$ having Euclidean radius $r=\tanh (\infty)=1_{X}$. In this realization, Lobachevskian space is viewed as the interior of a 3-dim ball $B^{3}\left(0,1_{X}\right)$ with its boundary at infinity represented by the 2-dimensional sphere $S^{2}\left(0,1_{X}\right)$.

The reader should note that the real (un-normalized) value of $\mathbf{1}_{X}$ (per astrophysical evidence) when compared with our geometric experience is incredibly high. It follows that on local distances, in all means, we experience Loba- 
chevskian 3-dim space as Euclidean 3-dim space. "All means" above should be understood as: our experimental techniques are unable to detect any departures from theorems of Euclidean geometry (e.g. the Pythagorean theorem) at distances up to about $10^{6}$ light years - ten times the diameter of Milky Way $\left(\sim 10^{5}\right.$ light years). Moving from a local to a global point of view, the entire Lobachevskian space $L_{X}^{3}$ is assembled from 3-dimensional Euclidean "patches" glued together with a mathematical object called a connection; however, we will not need to use this construction in the text.

In homogeneous spaces, loosely speaking, the neighborhood of any point $x \in L_{X}^{3} \quad$ "looks the same" as the neighborhood of any other point. In the other words all points of $L^{3}$ are equivalent, and are related by the equivalence relation $\xi R \xi^{\prime}$, executed by Lorentz $S L(2 C)$ group of motion $\xi=g \xi^{\prime}$ or $\xi=g^{*} \xi^{\prime} g$.

We say that the homogeneous space $L^{3}$ consists only of one orbit of any arbitrary point. It follows that physical processes in homogeneous spaces do not depend on a particular location since all points and their neighborhoods are "the same", or more precisely, equivalent. This is interpreted in physics as a postulate of no preferred frame. Homogeneous space(s) are very convenient since any process (properties) can be studied in the neighborhood of its neutral element (the "center" of homogeneous space) and then translated to any location by the group action.

In our discussion, in order to connect to experimental data, distances in Lobachevskian space $L_{X}^{3}$ need to be mapped onto distances in the relevant model of $L_{X}^{3}$ in Euclidean space. There are infinitely many such maps. We will use two maps which map internal Lobachevskian distances $l$ (and functions of those distances, e.g. kinetic energy) from Lobachevskian position space $L_{X}^{3}$ onto distances $d$ or $\delta$ in the Euclidean space $E$. Note that Lobachevskian distance $l$ is the only two-point invariant in Lobachevskian space with respect to Lorentz group $S L(2 C)$ action.

In the Beltrami - Klein model, distances $d$ and $l$ are related as:

$$
d=\tanh l
$$

In the Poincare model, distances $\delta$ and $l$ are related as:

$$
\delta=\tanh \frac{l}{2}
$$

A prompt calculation of the relation between Euclidean distances (Euclidean images) $d$ and $\delta$ (of Lobachevskian (hyperbolic) distance $l$ ) from Formulas (37) and (38) gives the following equation:

$$
d=\frac{2 \delta}{1+\delta^{2}}
$$

Formula (39) above represents a well known, explicit isomorphism between the Poincare and Beltrami-Klein models of Lobachevskian geometry [12]. All of the above also applies to the representation of Lobachevskian geometry via position space $L_{X}^{3}$, or to the representation of Lobachevskian space via velocity 
space $L_{U}^{3}$, which we discuss next.

\subsection{Representation of Lobachevskian Geometry as a 3-Dim Velocity/Momentum Space $L_{U}^{3}=S L(2 C) / S U(2)$}

The geometric properties of Lobachevskian real, 3-dim velocity/momentum space are precisely the same as the geometric properties of Lobachevskian 3-dim position space discussed above. However we elaborate on it a bit more.

Velocity space is regarded as a 3-dim real Lobachevskian (hyperbolic) space $L_{U}^{3}$. The Gaussian (negative) curvature has a value of $K=-c^{-2}$, where the constant $c>0, c \in R$, is regarded as the velocity of light in a vacuum. The generic value of $K$ is set to -1 , which translates to a choice of physical units in which $c=1$. The signed Lobachevskian distance $\theta$ between two points $p_{1}, p_{2} \in L_{U}^{3}$, $\pm \theta\left(p_{1}, p_{2}\right)$ is interpreted as relative Lobachevskian velocity $\theta$. Note that $\theta$ is in fact dimensionless. This is because the actual distance is $\theta \sqrt{-K}$, but since we set $K=-1$, the curvature $K$ is not present numerically. Curvature is of dimension inverse-squared-length, which makes the product $\theta \sqrt{-K}$ a pure real number with no physical label. Note also that the distance $|\theta|$, the relative velocity, is the only invariant between two points (two frames in relative uniform motion), with the respect to $S L(2 C)$ isometries in Lobachevskian space.

In a typical experimental High Energy Physics (HEP) experimental situation Lobachevskian velocities have to be mapped onto Euclidean velocities in the Euclidean space we live in. Depending on the choice of the map, the relation between Euclidean (relative) velocities $\mathrm{v}$, the velocities we measure experimentally $v$, and the internal Lobachevskian (hyperbolic) relative velocity $\theta$, are given by Formulas (40) and (41) below:

$$
\mathrm{v}=\tanh \theta
$$

due to the Beltrami-Klein model (shown in Section 4 to correspond to ESR), and

$$
v=\tanh \frac{\theta}{2}
$$

due to the Poincare model (shown in Section 5 to correspond to BSR). The isomorphism between the two maps (40) and (41) (the Beltrami-Klein and Poincare models) is given by:

$$
\mathrm{v}=\frac{2 v}{1+v^{2}}
$$

It is straightforward to see that in the case of Lobachevskian velocity/momentum space, $L_{U}^{3}$, the isomorphism between the Beltrami-Klein and the Poincare models of Lobachevskian geometry is related to the homotopy between a gnomonic and a stereographic map discussed in Section 2.

The internal Lobachevskian relative velocity $\theta$ can take any real values $\theta \in[0, \infty)$. In physics, maps (40) and (41), $\theta \rightarrow \mathrm{v}$, or $\theta \rightarrow v$ map the non-compact half line $[0, \infty)$ onto segment $[0,1)$. In applications to physics, 
in order to incorporate velocities of photons at the boundary at infinity, $\partial L_{U}^{3}(\infty)$ is added to Lobachevskian $L_{U}^{3}$ space, resulting in the so called extended Lobachevskian space $L_{U}^{3} \cup \partial L_{U}^{3}$. This way, $0 \rightarrow 0$, and $\infty \rightarrow c=1$. Relations $\theta+\infty=\infty$ and $\infty+\infty=\infty$, under maps (40) and/or (41) become $\mathrm{v}+c=c, v+c=c$, and $c+c=c$, or symbolically " $1+1=1$ ", which says that the velocity of light does not depend on the state of motion of a source (Michelson-Morley experiment), and that speed of light is the maximum speed possible.

Points $u$ in Lobachevskian 3 -dim velocity space are represented by $2 \times 2$ complex Hermitian positive definite matrices, $\tilde{u}=\left(\begin{array}{ll}u_{0}-u_{3} & u_{1}-i u_{2} \\ u_{1}+i u_{2} & u_{0}+u_{3}\end{array}\right)$. The set of complex $2 \times 2$ positive definite Hermitian matrices is not a group since the product of two such a matrices not need to be a positive definite matrix.

Note that the determinant of matrix $\tilde{u}$ is equal to $1, \operatorname{det} \tilde{u}=1$, which means that as a topological space, the Lobachevskian velocity space, in homogeneous coordinates $u\left(u_{1}, u_{2}, u_{3}, u_{4}\right)$, is modeled in a unit $(c=1)$ hyperboloid $[u, u]=u_{0}^{2}-u_{1}^{2}-u_{2}^{2}-u_{3}^{2}=1, u_{0} \geq 1$.

Remark 12 Since $[u, u]=1$ is equivalent to $\operatorname{det} \tilde{u}=1$, only three components of velocity $u$ are independent and consequently there is no such thing in nature as 4-velocity. Representation of $u$ via four homogeneous (projective) coordinates $u_{\alpha}, \alpha=0,1,2,3$ is merely a mathematical tool of convenience.

The representation of velocity space as a Lobachevskian space has the following properties:

1) Points at a finite Lobachevskian distance, $\theta<\infty$, from any internal point $u \in L_{U}^{3}$ represent velocities of massive $m_{0}>0$ particles.

2) Points at an infinite Lobachevskian distance, $\theta=\infty$, from any internal point $u \in L_{U}^{3}$ belong to the boundary at infinity $\partial L_{U}^{3}(\infty)$. They are interpreted as velocities of massless $m_{0}=0$ particles - photons (and perhaps neutrinos).

3) Space beyond the boundary at infinity is called imaginary Lobachevskian space. Its model is the one-sheet hyperboloid. Points beyond the boundary at infinity $\partial L_{U}^{3}(\infty)$ are thought as velocities of (so far) hypothetical particles - tachions. Distances in imaginary Lobachevskian space need not be real numbers.

At the end of this section, the reader is encouraged to remember that:

1) Lorentz group is the group $S L(2 C)$

2) Lobachevskian (hyperbolic) real $3 \mathrm{dim}$ space is a coset space of Lorentz $S L(2 C)$ group with respect to $S U(2) . L^{3}=S L(2 C) / S U(2)$.

3) Lobachevskian space $S L(2 C) / S U(2)$, in our particular discussion, has two isomorphic representations: one, as a coordinate position space $L_{X}^{3}=S L(2 C) / S U(2)$, and two, as a velocity/momentum space $L_{V(P)}^{3}=S L(2 C) / S U(2)$.

4) Lorentz group $S L(2 C)$ acts via isometries given by Formula (31) or (32) on either representation of $L^{3}$. 


\section{4. "Special Relativities" as Maps from Lobachevskian Space into Euclidean Space}

We have arrived at the point when we are ready to present "special relativities" and their true meaning as parametric maps. However, before we present our version of "special relativity", BSR, it will be beneficial for the reader to first see how the above mathematical exposition produces Einstein's "special relativity", ESR. Since the methodology applied to obtain either ESR or BSR is identical in both cases, the reader should gain confidence that the $S L(2 C)$ group action approach works and produces viable physics. So this section on ESR may be regarded as mini "warm up" before the next section on BSR.

\subsection{Einstein's "Special Relativity" (ESR) as a Gnomonic Map}

In this section, we present how we understand Einstein's “special relativity" (ESR) as a kind of a map, namely a gnonomic map. The reader will have the opportunity to compare our exposition of Einstein's ESR with an orthodox treatment via Minkowski flat 4-geometry as is commonly seen in the literature and to make his/her own conclusions.

Einstein's "special relativity" results from the left-action of the Lorentz Group $S L(2 C)$ on the Lobachevskian coordinate space $L_{X}^{3}=S L(2 C) / S U(2)$ :

$$
h=g h^{\prime}, h, h^{\prime} \in L_{X}^{3}, g \in S L(2 C)
$$

Remark 13 The action (43) has simple meaning for experimental high energy physics and astrophysics. It describes a single sided motion typical for experiments on accelerators with fixed target, e.g. Stanford's Linear Accelerator.

To extract some physics of interest from (43), we proceed as follows. The positive definite $2 \times 2$ Hermitian matrices $\tilde{x}, \tilde{x}^{\prime}$ represent points in Lobachevskian space $L_{X}^{3}=S L(2 C) / S U(2)$, and the $S L(2 C)$ matrix $g=\tilde{u}=\left(\begin{array}{cc}u_{0}-u_{3} & u_{2}-i u_{1} \\ u_{2}+i u_{1} & u_{0}+u_{3}\end{array}\right)$, viewed as a Hermitian operator, represents the motion operator, which sends the point $\tilde{x}^{\prime}$ onto point $\tilde{x}$. Therefore we come to the following matrix equation:

$$
\tilde{x}=\tilde{u} \tilde{x}^{\prime}, \tilde{x}, \tilde{x}^{\prime} \in L_{X}^{3}=S L(2 C) / S U(2), \tilde{u} \in S L(2 C),
$$

The simple matrix equation $\tilde{x}=\tilde{u} \tilde{x}^{\prime}$ (44) contains the entirety of Einstein's "special relativity" in the sense that all of ESR can be reproduced from it. Mathematically it is a left translation (isometry) on the coordinate Lobachevskian space executed by the motion operator $\tilde{u}$. From the point of view of physics, it relates two points $\tilde{x}^{\prime}$ and $\tilde{x}$ in Lobachevskian position space (hyperboloid) which are in relative uniform motion represented by the operator $\tilde{u}$ acting on Lobachevskian space - position hyperboloid $\operatorname{det} \tilde{x}=1=x^{2}$. The coordinates of both points $x$ and $x^{\prime}$ are projective (homogeneous) coordinates in Lobachevskian position space. That is the essence of ESR.

For reasons of simplicity alone, we represent $2 \times 2$ matrices $\tilde{u}$ in diagonal form, which from the point of view of physics, means that the velocity $u$ is con- 
fined to the $x_{0}, x_{3}$ plane and consequently has only two $u_{0}, u_{3}$ homogeneous components. In non-homogeneous affine coordinates $\frac{u_{3}}{u_{0}}=\mathrm{v}_{3}$, relative velocity has only one component $\mathrm{v}_{3}$.

Remark 14 Note that in the one-dimensional model we are analyzing, the complex Lorentz group $S L(2 C)$ reduces to a group of real diagonal matrices, meaning the subgroup of $S L(2 R)$ - the Lorentz group of real $2 \times 2$ matrices with unit determinant. Note also that in the diagonal representation the matrix operator is in fact expressed by its eigenvalues, which we will calculate explicitly in Section 4.1.3.

Equation (44) now yields:

$$
\left(\begin{array}{cc}
x_{0}-x_{3} & 0 \\
0 & x_{0}+x_{3}
\end{array}\right)=\left(\begin{array}{cc}
u_{0}-u_{3} & 0 \\
0 & u_{0}+u_{3}
\end{array}\right)\left(\begin{array}{cc}
x_{0}^{\prime}-x_{3}^{\prime} & 0 \\
0 & x_{0}^{\prime}+x_{3}^{\prime}
\end{array}\right)
$$

We can obtain the transformed $x_{0}$ coordinate from the trace property, observing that for diagonal matrices $a, b, c$, if $a=b c \Rightarrow \operatorname{Tr} a=\operatorname{Tr}(b c)$. We obtain the transformed coordinate $x_{3}$ by comparing the appropriate matrix elements in both sides of (45) and then subtracting them. This gives:

$$
\begin{aligned}
& x_{0}=u_{0} x_{0}^{\prime}+u_{3} x_{3}^{\prime} \\
& x_{3}=u_{3} x_{0}^{\prime}+u_{0} x_{3}^{\prime}
\end{aligned}
$$

Or in the matrix form:

$$
\left(\begin{array}{l}
x_{0} \\
x_{3}
\end{array}\right)=\left(\begin{array}{ll}
u_{0} & u_{3} \\
u_{3} & u_{0}
\end{array}\right)\left(\begin{array}{l}
x_{0}^{\prime} \\
x_{3}^{\prime}
\end{array}\right)
$$

Remark 15 For $U=\left(\begin{array}{ll}u_{0} & u_{3} \\ u_{3} & u_{0}\end{array}\right), U^{-1}=\left(\begin{array}{cc}u_{0} & -u_{3} \\ -u_{3} & u_{0}\end{array}\right)$. Thus if $x=U x^{\prime}$, then $U^{-1} x=x^{\prime}$. From the point of view of physics, the velocity sign is reversed in the inverse matrix $U^{-1}$. This obviously means that if the system $A$ ' is in motion with velocity $V$, with respect to the system $A$, then $A$ is moving with velocity $-V$, with respect to $A^{\prime}$.

We recall that in Lobachevskian velocity space $u_{0}^{2}-u_{3}^{2}=1=u_{0}^{2}\left(1-\frac{u_{3}^{2}}{u_{0}^{2}}\right) \Rightarrow$ $u_{0}=\frac{1}{\sqrt{1-v_{3}^{2}}}$. The non-homogeneous (local) coordinates are related to projective (homogeneous) coordinates as: $\mathrm{v}_{3}=\frac{u_{3}}{u_{0}}$. Velocity is aligned along $u_{3}$, and in non-homogeneous coordinates relative velocity $\mathrm{v}$ has only one component.

If we regard the projective coordinate $x_{0}$ as "time" (measured in meters of a light-path), then Equations (46) and (47) can be rewritten in terms of a temporal $x_{0}$ and a spatial coordinate $x$.

Now we abandon subscripts, and keeping in mind that the normalization $c=1$ affects only physical units and that $x_{0}=c t$, we end up with the well know formulas from Einstein's "special relativity" for temporal and spatial coordinate transformation in systems being in uniform relative motion: 


$$
\begin{aligned}
& t=\frac{t^{\prime}+\mathrm{v} x^{\prime}}{\sqrt{1-\mathrm{v}^{2}}} \\
& x=\frac{\mathrm{v} t^{\prime}+x^{\prime}}{\sqrt{1-\mathrm{v}^{2}}}
\end{aligned}
$$

Equations (49) and (50) were introduced by Albert Einstein in 1905 in his work [13], however due an entirely different form of reason from our own.

In the Euclidean limit, Equations (49) and (50) are:

$$
\begin{gathered}
t=t^{\prime} \\
x=\mathrm{v} t+x^{\prime}
\end{gathered}
$$

Equations (51) and (52) are known as Galilean transformations due to single sided motion. In physics they correspond to fixed target scattering, like in the Stanford Linear Accelerator.

Transformations (49) and (50) in compact form are:

$$
\left(\begin{array}{l}
t \\
x
\end{array}\right)=\left(\begin{array}{cc}
\frac{1}{\sqrt{1-\mathrm{v}^{2}}} & \frac{\mathrm{v}}{\sqrt{1-\mathrm{v}^{2}}} \\
\frac{\mathrm{v}}{\sqrt{1-\mathrm{v}^{2}}} & x \frac{1}{\sqrt{1-\mathrm{v}^{2}}}
\end{array}\right)\left(\begin{array}{l}
t^{\prime} \\
x^{\prime}
\end{array}\right)
$$

Here relative velocity $\mathrm{v}$ is in fractions of the velocity of light $c$. If $c$ has to be present explicitly, then we need to re-scale: $\mathrm{v} \rightarrow \frac{\mathrm{v}}{c}$ and substitute $c t$ for $x_{0}$.

Since the matrix in (53) is uni-modular, there is a unique parametrization of the matrix elements as:

$$
\begin{gathered}
\cosh \theta=\frac{1}{\sqrt{1-\mathrm{v}^{2}}}, \sinh \theta=\frac{\mathrm{v}}{\sqrt{1-\mathrm{v}^{2}}} \\
u_{0}=\frac{1}{\sqrt{1-\mathrm{v}^{2}}}, \quad u_{i}=\frac{\mathrm{v}_{i}}{\sqrt{1-\mathrm{v}^{2}}}, \quad i=1,2,3,|\mathrm{v}|<1
\end{gathered}
$$

The coordinates (55) are called Weierstrass coordinates due to the German mathematician Weierstrass [8]. Weierstrass coordinates were already discussed in Section 2 and the reader is encouraged to review. It is easy to see that Weierstrass homogeneous coordinates (55) are normalized to unity $u^{2}=u_{0}^{2}-u_{1}^{2}-u_{2}^{2}-u_{0}^{2}=1$. Weierstrass used coordinates (55) in his work on Lobachevskian (hyperbolic) geometry more than 50 years before ESR, however, Einstein never mentioned or referred to them.

From Formula (54) we conclude that the internal Lobachevskian velocity $\theta$ and local Euclidean velocity $\mathbf{v}$ measured in experiments are related by:

$$
\tanh \theta=\mathrm{v}
$$

which is the equation by which distances in Lobachevskian space are mapped onto Euclidean distances due to the Beltrami-Klein model, here representing Lobachevskian space by the space of velocities $L_{U}^{3}$. It should be noted that $\theta$, the internal Lobachevskian velocity is incorrectly (in our opinion) called the "hyperbolic angle" or "rapidity" in the literature on high energy particle physics. 
Conclusion 16 Comparing Formulas (55) and (56) resulting from Lorentz transformations (in Einstein's "special relativity") with Formulas (14) and (17) which we derived from the gnomonic (central) projection from hyperboloids, we see that they are identical. This means Einstein's "special relativity" is nothing more than a gnomonic map from Lobachevskian space into Euclidean space. This is the true meaning of ESR.

\subsubsection{Addition of Velocities along the Same Direction}

From the map (56) between Lobachevskian and Euclidean velocities we easily see that:

$$
\tanh \left(\theta_{1}+\theta_{2}\right)=\frac{\tanh \theta_{1}+\tanh \theta_{2}}{1+\tan \theta_{1} \tanh \theta_{2}}=\frac{\mathrm{v}_{1}+\mathrm{v}_{2}}{1+\mathrm{v}_{1} \mathrm{v}_{2}}
$$

Recall that $\theta$ in (57) is not an angle. It is a real non-negative number $\theta \in(0, \infty)$ representing the unit-less length of a Lobachevskian linear segment in 1-dim Lobachevskian space, i.e. a Lobachevskian line. A Lobachevskian line is a one dimensional, real, metric, non-compact space of constant negative curvature. An image of $\theta$ in Euclidean space is what is measured by physicists due to various maps; in this case due to the Beltrami-Klein map, $\mathrm{v}=\tanh \theta$. It seems that at the time he introduced ESR, Einstein was not familiar with the work of Lobachevski on non-Euclidean geometry; nevertheless he deduced the correct formula for addition of velocities.

\subsubsection{Distortions}

From Equations (49) and (50) we can obtain the distortions resulting from Einstein's ESR map. These distortions, due to historical reasons, are called "time dilation" and "length contraction", or "Fitzerald contraction".

$$
\begin{aligned}
& \Delta t=\Delta t^{\prime} \frac{1}{\sqrt{1-\mathrm{v}^{2}}}=\Delta t^{\prime} \cosh \theta \\
& \Delta x=\Delta x^{\prime} \frac{1}{\sqrt{1-\mathrm{v}^{2}}}=\Delta x^{\prime} \cosh \theta
\end{aligned}
$$

From (58) and (59), we see that when the curvature approaches zero, $-K=c^{-2} \rightarrow 0$ equivalent to $\mathrm{v}<<c$ (Euclidean maps), the apparent distortions vanish and $\Delta t=\Delta t^{\prime}, \Delta x=\Delta x^{\prime}$.

\subsubsection{Physical Meaning of Diagonal Entries (Eigenvalues) of Motion Operator $\tilde{\boldsymbol{u}}$}

To see the physical meaning of the diagonal entries $\Lambda_{1}=u_{0}-u_{3}$ and $\Lambda_{2}=\Lambda_{1}^{-1}=u_{0}+u_{3}$ of the motion operator $\tilde{u}$ we need to express them in local coordinates $\frac{u}{u_{o}}$. Using Weierstrass coordinates for $u$ yields:

$$
\Lambda_{1}=\sqrt{\frac{1+\mathrm{v}}{1-\mathrm{v}}}, \quad \Lambda_{2}=\Lambda_{1}^{-1}=\sqrt{\frac{1-\mathrm{v}}{1+\mathrm{v}}}
$$

Thus the diagonal entries of the motion operator are easily recognized as Lobachevski - Doppler blue $\Lambda_{1}$ and red $\Lambda_{2}$ frequency shifts for a single sided 
motion expressed in local coordinates v. Elements of $S L(2 R)$ with reciprocal values like in (60) in the geometry of the Mobius group are called hyperbolic transformations.

\subsection{Lobachevski - Poincare - Von Brzeski "Special Relativity" (BSR) as a Stereographic Map}

In this section, we show that our version of special relativity, i.e. BSR, is a stereographic projection from Lobachevskian space (a hyperboloid) discussed in Section 2.2.2. A stereographic projection from a hyperboloid results in the Poincare representation of Lobachevskian geometry in the unit ball, or in our case, in the unit disc.

As we discussed in Section 3, the Lorentz group may act on Lobachevskian space $L_{X}^{3}$ in several ways. Our choice is the action (33) via the automorphism $h=g^{*} h^{\prime} g$ of $L_{X}^{3}$ executed by a double sided motion (double sided translation), which we now compute in detail.

Remark 17 The action (32) is of direct and fundamental significance for experimental high energy physics. It represents a typical case of center of momentum scattering of identical particles in accelerators with counter rotating beams, see Figure 6.

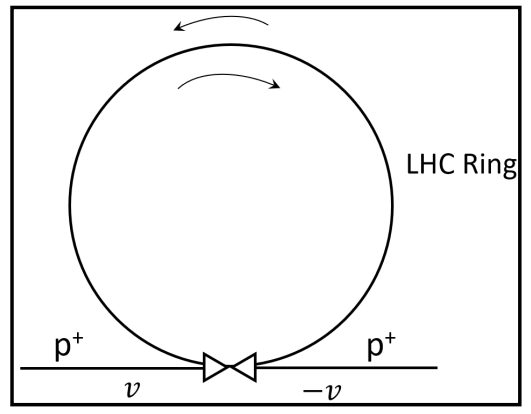

Figure 6. Two protons with opposite velocities $v$ and $-v$ collide in the LHC ring.

The point $\tilde{x}$ in Lobachevskian space $L_{X}^{3}$ is represented by a uni-modular positive definite Hermitian matrix

$$
\tilde{x}=\left(\begin{array}{ll}
x_{0}-x_{3} & x_{2}-i x_{1} \\
x_{2}+i x_{1} & x_{0}+x_{3}
\end{array}\right)
$$
$\tilde{x} \in L^{3}=H^{+}$and for matrix $g=\left(\begin{array}{ll}\alpha & \beta \\ \gamma & \delta\end{array}\right)$ in (32) we take, as in Section 3, the
Hermitian motion operator matrix:

$$
\tilde{u}=\left(\begin{array}{ll}
u_{0}-u_{3} & u_{2}-i u_{1} \\
u_{2}+i u_{1} & u_{0}+u_{3}
\end{array}\right)
$$

We see that $\operatorname{det} \tilde{u}=u^{2}=u_{0}^{2}-u_{1}^{2}-u_{2}^{2}-u_{3}^{2}=1$, which tells us that if $u_{0} \geq 1$, the point $u$ belongs to the upper sheet the of hyperboloid $u^{2}=1$.

Substituting in $h=\tilde{x}, h^{\prime}=\tilde{x}^{\prime}$, and $g=\tilde{u}^{\prime}$ into Equation (32) yields:

$$
\tilde{x}=\tilde{u}^{*} \tilde{x}^{\prime} \tilde{u}
$$

As we have already said, matrices $\tilde{u}$ and $-\tilde{u}$ correspond to the same 
transformation and are liable for identification.

The action (63) leaves the quadratic form

$Q(x, x)=Q\left(x^{\prime}, x^{\prime}\right)=\operatorname{det} \tilde{x}^{\prime}=\operatorname{det} \tilde{x}=x^{2}=x^{\prime 2}=1$ invariant, which is due to the invariance of the matrix determinant det $\tilde{x}$ under transformations (63). Geometrically, this means that the hyperboloid $x^{\prime 2}=1$ is invariant (is mapped onto itself) under all motions (63). This simple matrix Equation (63), $\tilde{x}=\tilde{u}^{*} \tilde{x}^{\prime} \tilde{u}$, contains the entirety of the authors' "special relativity" BSR, in the sense that BSR can be derived from it.

For the purposes of the present work, just for simplicity, we will limit ourselves to one dimension. One dimensional Lobachevskian space or the Lobachevskian line has two homogeneous (projective) coordinates $x_{0}$ and $x_{3}$. The Lobachevskian line is aligned along $x_{3}$. Equation (63) in the one dimensional case is as follows. We write the coordinates $x=x\left(x^{\prime}\right)$, due to motion (63) in $L_{X}^{1}$ executed by $g \in S L(2 C)$ i.e. the Lorentz transformations. In one dimensional space, the matrices (61) and (62) become diagonal which greatly simplifies the calculations.

Since in the one dimensional case, velocity $u$ and displacement $x$ are aligned along the same line, the matrices (61) and (62) now are real diagonal and hence commutative.

The one dimensional motion operator is now:

$$
\tilde{u}=\left(\begin{array}{cc}
u_{0}-u_{3} & 0 \\
0 & u_{0}+u_{3}
\end{array}\right), \quad \tilde{u} \in S L(2 C)
$$

acting on one dimensional Lobachevskian space $L_{X}^{1}$ :

$$
\tilde{x}=\left(\begin{array}{cc}
x_{0}-x_{3} & 0 \\
0 & x_{0}+x_{3}
\end{array}\right), \quad \tilde{x} \in S L(2 C) / S U(2)
$$

according to the double sided action as:

$$
\tilde{x}=\left(\begin{array}{cc}
u_{0}-u_{3} & 0 \\
0 & u_{0}+u_{3}
\end{array}\right)\left(\begin{array}{cc}
x_{0}^{\prime}-x_{3}^{\prime} & 0 \\
0 & x_{0}^{\prime}+x_{3}^{\prime}
\end{array}\right)\left(\begin{array}{cc}
u_{0}-u_{3} & 0 \\
0 & u_{0}+u_{3}
\end{array}\right)
$$

We can compute the double sided action (66) instantly by observing that for $2 \times 2$ diagonal matrices $a, b, c, d$, if $a=b c d$ then $\operatorname{Tr}(a)=\operatorname{Tr}(b c d)$, and from (66) we find that:

$$
\begin{aligned}
& x_{0}=\left(u_{0}^{2}+u_{3}^{2}\right) x_{0}^{\prime}+2 u_{0} u_{3} x_{3}^{\prime} \\
& x_{3}=2 u_{0} u_{3} x_{0}^{\prime}+\left(u_{0}^{2}+u_{3}^{2}\right) x_{3}^{\prime}
\end{aligned}
$$

We write transformations (67) and (68) in a slightly different form by converting homogeneous $u$ velocity into affine $v$ velocity which we measure in experiments. Recall that in homogeneous coordinates in Lobachevskian space $u^{2}=1$, and $1=u_{0}^{2}\left(1-v \frac{u_{3}^{2}}{u_{0}^{2}}\right)$. In homogeneous coordinates $v_{3}$ is: $v_{3}=\frac{u_{3}}{u_{0}}$. It follows that,

$$
u_{0}^{2}+u_{3}^{2}=\frac{1+v_{3}^{2}}{1-v_{3}^{2}}
$$




$$
u_{0} u_{3}=\frac{v_{3}}{1-v_{3}^{2}}
$$

Therefore, the transformations of homogeneous coordinates on a Lobachevskian line due to two sided translations are:

$$
\begin{aligned}
& x_{0}=\frac{\left(1+v^{2}\right) x_{0}^{\prime}+2 v x_{3}^{\prime}}{1-v^{2}} \\
& x_{3}=\frac{2 v x_{0}^{\prime}+\left(1+v^{2}\right) x_{3}^{\prime}}{1-v^{2}}
\end{aligned}
$$

Therefore, if we assume that the projective zero coordinate $x_{0}$ in (71) and (72) is understood as "time" ct (measured in meters of a light-path) then taking $c=1$ we can relabel $x_{0}=t$ and $x_{0}^{\prime}=t^{\prime}$, we arrive at the following equations:

$$
\begin{aligned}
& t=\frac{\left(1+v^{2}\right) t^{\prime}+2 v x^{\prime}}{1-v^{2}} \\
& x=\frac{2 v t^{\prime}+\left(1+v^{2}\right) x^{\prime}}{1-v^{2}}
\end{aligned}
$$

Equations (73) and (74) are authors' transformations for temporal $t$ and spatial coordinates $x$ in systems being in relative uniform motion. The Equations (73) and (74) of the authors' "special relativity" (BSR) are an alternative to Einstein's transformations (49) and (50) in his ESR.

The reader should note that from the point of view of physics, our transformations correspond to the center of momentum frame, which is the bread and butter of particle scattering experiments for identical particles in circular accelerators with counter-rotating beams. The reader should also be aware that in (73) and (74), the velocity $|v| \in[0,1)$ is dimensionless, and that the speed of light factor $c=1$ is not present explicitly and it only affects physical units. To get (73) and (74) in common physical units, substitute $v \rightarrow \frac{v}{c}$ and $x_{0} \rightarrow c t$.

In the Euclidean limit, transformations $(73)$ and $(74)$ yield:

$$
\begin{gathered}
t=t^{\prime} \\
x=2 v t+x^{\prime}
\end{gathered}
$$

Remark 18 We'd like to explain the term "Euclidean limit" used above to linearize (74) and (75). In common MKS units, for example, the term $2 v x$ in the numerator of (74) is $2 \frac{v}{c} \frac{x}{c}$ and it is on the order of $c^{-2}$. We note that the Gaussian curvature of Lobachevskian velocity space is $K=-c^{-2}$. Therefore, we are neglecting the curvature of Lobachevskian space. Neglecting the curvature of Lobachevskian geometry implies that locally (in local coordinates $v$ ), the geometry can be approximated by Euclidean geometry to an arbitrary degree of precision [13]. In other words, we use the fact that around any of its points, velocity space is approximately flat (Euclidean). The procedure itself is called linearization or Euclideanization of physics modeled on curved, non-Euclidean, 
geometries.

Transformations (73) and (74) can be written in a compact form:

$$
\left(\begin{array}{l}
t \\
x
\end{array}\right)=\left(\begin{array}{cc}
\frac{1+v^{2}}{1-v^{2}} & \frac{2 v}{1-v^{2}} \\
\frac{2 v}{1-v^{2}} & \frac{1+v^{2}}{1-v^{2}}
\end{array}\right)\left(\begin{array}{l}
t^{\prime} \\
x^{\prime}
\end{array}\right)
$$

A quick look at entries of a matrix (77) tells us that in Lobachevskian velocity space $L_{U}^{3}$, we use projective (homogeneous) coordinates $u\left(u_{0}, u_{1}, u_{2}, u_{3}\right)$ of the form already introduced in Section 2 for stereographic projection from Lobachevskian space.

$$
u_{0}=\frac{1+v^{2}}{1-v^{2}}, u_{i}=\frac{2 v}{1-v^{2}}, i=1,2,3,|v|<1
$$

normalized such that $u^{2}=u_{0}^{2}-u_{1}^{2}-u_{2}^{2}-u_{3}^{2}=1$. Note that our projective (homogeneous) coordinates are different from Weierstrass projective (homogeneous) coordinates we encountered in Einstein's ESR, which should be no surprise since ours and Einstein's "relativity" refer to different models of Lobachevskian velocity space, namely Poincare and Beltrami-Klein, respectively.

Since the matrix in (77) is unimodular, $\operatorname{det}()=$.1 , there is a unique real parameter $\theta$ such that:

$$
\frac{1+v^{2}}{1-v^{2}}=\cosh \theta=u_{0}
$$

and

$$
\frac{2 v}{1-v^{2}}=\sinh \theta=u
$$

From (79) and (80) we find that:

$$
\tanh \theta=\frac{2 v}{1+v^{2}}
$$

which is the isomorphism between the Poincare and the Beltrami-Klein models of Lobachevskian geometry, in (27) and (28), we mentioned in Section 2.2.2.

Since $\tanh \frac{\theta}{2}=\left(\frac{\cosh \theta-1}{\cosh \theta+1}\right)^{\frac{1}{2}}$, using (79) and (80) we can find $v$ versus $\theta$ :

$$
v=\tanh \frac{\theta}{2}
$$

Formula (82) is the map of Lobachevskian velocity $\theta$ onto local Euclidean velocity $v$ due to the Poincare model of Lobachevskian geometry. The parameter $\theta$ in (82) is the intrinsic Lobachevskian, dimensionless, relative velocity $|\theta| \in[0,+\infty)$. In particle physics it is often (in our view) incorrectly called the "velocity parameter" or "rapidity". Velocity $|v| \in[0,1)$ is the image of Lobachevskian velocity $\theta$, as it appears to us, in Euclidean space. Note that $\theta$ is dimensionless because the Gaussian curvature of Lobachevskian velocity space is set to $K=-1$. The function $\tanh ($.$) relates Lobachevskian and Euclidean dis-$ 
tances. The value $\theta=\infty$ and $v=1$ is restricted to photons (and perhaps to neutrinos) which are points at the boundary at infinity for Lobachevskian velocity space.

Comparing BSR Formulas (79) and (80) resulting from the $S L(2 C)$ Lorentz group action (Lorentz transformations) to Formulas (19) and (20) resulting from Lobachevskian cartography in the case of a stereographic projection from hyperboloids, we see that seemingly unrelated areas of science, cartography and "special relativity" are in fact two sides of the same coin. Both are just certain maps of infinitely many possible maps (as we will see below).

Conclusion 19 The image of the double sided action (63) of the Lorentz group $S L(2 C)$, or the image of the double sided motion, on a real 3-dim Lobachevskian space $S L(2 C) / S U(2)$ (viewed as a hyperboloid $x^{2}=1$ ), is isomorphic with the Poincare ball model representation of Lobachevskian space resulting from a stereographic projection of Lobachevskian space (hyperboloid) into Euclidean space; see Figure 5.

\subsubsection{Addition of Velocities along the Same Direction}

In accordance with (82) we have the following equation:

$$
\tanh \left(\frac{\theta_{1}}{2}+\frac{\theta_{2}}{2}\right)=\frac{\tanh \frac{\theta}{2}+\tanh \frac{\theta}{2}}{1+\tanh \frac{\theta}{2} \tanh \frac{\theta}{2}}=\frac{v_{1}+v_{2}}{1+v_{1} v_{2}}
$$

which is the same as in Einstein's ESR. This is a model independent result. Recall that $\theta$ in (83) is not an angle. It is a real non-negative number $\theta \in(0, \infty)$ representing a unit-less length of a Lobachevskian linear segment in one dimensional Lobachevskian space, i.e. a Lobachevskian line. As we mentioned above, a Lobachevskian line is one dimensional, real, metric, non-compact space of constant negative curvature. An image of $\theta$ in Euclidean space is measured by physicists due to various maps. In this case due to the Poincare map: $v=\tanh \frac{\theta}{2}$.

\subsubsection{Distortions of Maps in BSR and Their Geometric Meaning}

From the transformations laws or maps (73) and (74) it is easy to see that the apparent distortions for spatial and temporal intervals (for a two sided uniform motion, i.e. at fixed distance in Lobachevskian velocity space) in BSR, given by the diagonal terms of matrix (77), will be:

$$
\begin{aligned}
& \Delta t=\Delta t^{\prime} \frac{1+v^{2}}{1-v^{2}}, \text { or } \frac{\Delta t}{\Delta t^{\prime}}=\cosh \theta \\
& \Delta x=\Delta x^{\prime} \frac{1+v^{2}}{1-v^{2}}, \text { or } \frac{\Delta x}{\Delta x^{\prime}}=\cosh \theta
\end{aligned}
$$

Equations (84) and (85) are obviously distortions of a stereographic projection from a hyperboloid into in the Euclidean plane seen as the Poincare disc model.

The reader should note that the dimensionless velocity $v$ in (84) and (85) in 
the MKS system of units will be $\frac{v^{2}}{c^{2}}=v^{2} c^{-2}=v^{2}|K|$ where $-K=c^{-2}$ is the Gaussian curvature of Lobachevskian velocity space. Evidently, as $K \rightarrow 0$, and velocity space becomes Euclideanized, the distortions (84) and (85) of images under map (73) and (74) vanish and $\Delta t=\Delta t^{\prime}, \Delta x=\Delta x^{\prime \prime}$, meaning this is a case of a mapping between two flat spaces. Maps in such cases will be distortion-less. No apparent "time dilation" and no apparent "length contraction" will exist. In the literature, such maps are referred to as Galilean.

\subsubsection{Physical Meaning of Diagonal Entries (Eigenvalues) of Motion Operator $\tilde{\boldsymbol{u}}$}

It is easy to see that the diagonal entries of the double sided motion operator are:

$$
\begin{aligned}
& \Lambda_{1}=\frac{1+v}{1-v} \\
& \Lambda_{2}=\frac{1-v}{1+v}
\end{aligned}
$$

These are Lobachevski-Doppler blue (86) and red (87) shifts for two sided motion. Examples are:

1) A frequency shift from the reflection from a moving mirror recorded by a transceiver when both, transceiver and mirror (target) are in motion (e.g. when radar and target are both in flight).

2) Frequency shifts recorded in reflective telescopes.

3) Experiments in circular accelerators with counter rotating beams, see Figure 6.

\subsubsection{Practical Applications to Astrophysics of Formulas for Frequency Shifts in Beltrami-Klein (ESR) and in Poincare (BSR) Models}

Frequency shifts resulting from Lobachevskian geometry are given either by Formulas (60) in Beltrami-Klein representation of Lobachevskian geometry (ESR), or by Formulas (86) and (87) in the Poincare representation of Lobachevskian geometry (BSR). These formulas apply either to a Lobachevskian large scale vacuum resulting in cosmological redshift, or to a Lobachevskian velocity space resulting in known Doppler shift. Recall that both position and velocity Lobachevskian spaces are metric spaces. The signed distance in Lobachevskian velocity space is called relative velocity.

Since the formulas for frequency shifts are widely used in astrophysics, radar techniques, warfare, medicine, and in nuclear physics (e.g. Mossbauer effect), and since the numbers representing spectral shifts derived from those models differ, it is very relevant which "special relativity" Formulas (ESR or BSR) will be used to draw conclusions about the physics represented by the measured frequency shifts $z$. Figure 7 shows the uncertainties in conclusions due to ESR and BSR in a typical astrophysics scenario. We illustrate it via simplified three examples below.

Suppose, for instance, that the measured redshift is $z=2$ and let us analyze what this real number 2 tells us about physics. 


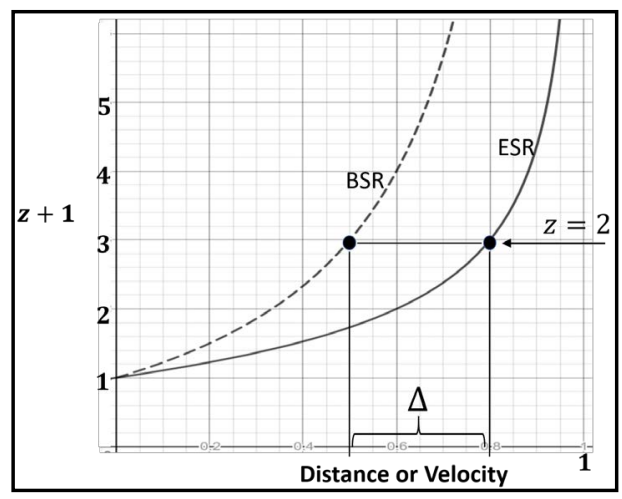

Figure 7. In the figure above, the dashed line shows the spectral shift $z$ due to BSR, and the solid line shows the spectral shift due to ESR. The horizontal axis is calibrated in units of distance in position space, $R=1=15$ billion light-years, in which case $\Delta=\Delta x$ refers to the difference in position due to BSR and ESR, or in units of distance in velocity space, $c=1=3 \times 10^{8} \mathrm{~m} / \mathrm{s}$, in which case $\Delta=\Delta u$ refers to the difference in velocity due to BSR and ESR.

- Example 1. Let us assume that the measured redshift $z=2$ from some source is a result of the distance in position space, meaning a negatively curved Lobachevskian Universe and there is no relative motion of the source with respect to observer. In this case, assuming arbitrarily that the radius of the Lobachevskian Universe regarded as an interior of Euclidean ball is say 15 billion light years (bly), normalized to $R=1$, we see in Figure 7, that applying ESR we obtain the distance to the source of $7.5 \mathrm{bly}=0.5 R$, while applying BSR we obtain a distance to the source $12 \mathrm{bly}=0.8 R$. We see that the uncertainty $\Delta_{X}$ in location of a luminous object is $\Delta_{X}=4.5$ bly.

- Example 2. Let's assume now that a luminous object is "close", so we neglect the spectral shift due to the distance in position space, and all of the redshift $z=2$ is due to distance in velocity space, i.e. relative ( receding ) velocity. We view Lobachevskian velocities space as an interior of unit radius $R=c=1$ equipped with hyperbolic metric. The signed distance here is simply relative velocity. In this case, the uncertainty in velocity determination $\Delta_{U}$ due to the different maps ESR and BSR is $\Delta_{U}=0.8 c-0.5 c=90000$ $\mathrm{km} / \mathrm{sec}$.

- Example 3. Taking into consideration both velocity and position, we come to the notion of a phase space. In the phase space picture the uncertainty of position and velocity is simply $\Delta_{X} \times \Delta_{U}$ and the only thing we can say is that an object is somewhere in an uncertainty cell of phase space having size $\Delta_{X} \times \Delta_{U}=4.5$ bly $\times 90,000 \mathrm{~km} / \mathrm{sec}$. The uncertainty relation $\Delta_{X} \times \Delta_{U}$ is quite close analogous to Heisenberg's uncertainty relation $\Delta x \times \Delta p$ in quantum mechanics.

If we recall that Lobachevskian geometry affects not only color of light (spectrum) but also its intensity, which makes Euclidean photometry non-applicable and misleading [4], we find ourselves in situation where the entire deep space astrophysics and cosmology cannot be trusted as providing viable picture of the 
Universe.

\subsection{Advantages and Generality of Our Approach}

The formalism presented here is intuitively simple and does not require more than three real dimensions. Recall that since $g \in S L(2 C)$, $\operatorname{det} \tilde{x}=1$, hyperboloid $x^{2}=$ const. $>0, x_{0} \geq 1$, is mapped onto itself under motions (31) or (32). It follows that the physical phenomena as described in Einstein's original ESR are due to isometries (rigid motions) within a Lobachevskian 3-dim real space. ESR does not extend to the entire $R^{4}$ but is restricted to only a Lobachevskian real 3-dim space (hyperboloid) $x^{2}=x_{0}^{2}-x_{1}^{2}-x_{2}^{2}-x_{3}^{2}=1, x_{0} \geq 0$, invariant under the action of the Lorentz group $S L(2 C)$. Hence, there is no point in paying attention to what is going on "outside" of Lobachevskian space $x^{2}=1$.

The main point which is totally missed in orthodox treatments of "special relativity" based on $S O(1,3)$ is the importance of the Lorentz group $S L(2 C)$ in physics, which is the group of isometries of real 3-dim Lobachevskian space, and it is the foundation of Lobachevskian geometry and Lobachevskian physics. Note that non-integer spinor fields (e.g. electron fields) are associated with the representations of $S L(2 C)$ instead of the (proper) Lorentz group $S O(1,3)$.

It is worth noting that our method is quite general. Take for example, a Lobachevskian plane regarded as the upper-half plane $\operatorname{Im}(z)>0$ in complex variables. Then the "Lorentz group" acting via isometries on the upper-half plane is the group of fractional linear transformations $z \rightarrow \frac{a z+b}{c z+d}$, which is the Mobius group, and we can obtain the transformation of "time" and position coordinates in yet another "special relativity" in this case.

Regarding ESR, the Minkowski treatment focuses on a pseudo-Euclidean (flat) embedding space instead on negatively curved Lobachevskian embedded space where physics happens. The Minkowski 4-dim pseudo-Euclidean interpretation of "special relativity" allows us to compute various entities via ESR in Minkowski's picture, but the reasons for physical mechanisms are entirely absent, paradoxes are present, and generalizations are impossible.

Obviously physics in a manifold does not depend on the ambient embedding space in which a manifold is embedded. An observer looking at a globe from the ambient three dimensional Euclidean space in his room will see a model airplane flying from San Francisco to Frankfurt along a path having three coordinates $x, y, z$ in flat three dimensional space. However, the autopilot which actually guides the plane from San Francisco to Frankfurt uses only two coordinates on the surface of Earth, longitude and latitude. The autopilot "thinks" exclusively in terms of a two dimensional (albeit curved) space, and knows nothing about the fact that the Earth's surface $S^{2}$, is embedded into some higher dimensional space $E^{3}$ because this information is entirely irrelevant. Studies of geometry and physics from the point of view of higher dimensional embedding spaces 
were typical for the 18th and 19th centuries. The modern approach is via internal geometry and internal physics in the embedded spaces.

The more relevant view on ESR, as a physical 3-dim Lobachevskian geometry, as pointed out by Barrett [14], was promoted by Vladimir Varicak, a Croatian mathematician and physicist in as early as 1908 [15]. Unfortunately, it appears that his papers were not understood by the scientific community and went mostly unnoticed. Physicists fascinated with 4-dim Minkowski flat ambient geometry were seemingly unable or unwilling to pay attention to Varicak's work. It is interesting to note that while developing and publishing his "special relativity" (ESR) [9] in 1905, Albert Einstein, influenced perhaps by his teacher Minkowski, focused his attention on the linear (pseudo-Euclidean) 4-dim space instead of directly mapping 3-dim Lobachevskian geometry (known since 1835) into a 3-dim Euclidean space. It is remarkable that a whole generation of physicists during the past $100+$ years has focused on Minkowski's embedding space, and has missed the very essence that "special relativity" (ESR) is just a gnomonic map and as such cannot be unique.

At this point it is worth reviewing what we have covered so far:

1) Lorentz transformations, induced by elements of the Lorentz group $S L(2 C)$, are isometries of a real 3-dim Lobachevskian (hyperbolic) space and as such are distortion-less.

2) Various parametric maps from real 3-dim Lobachevskian space into Euclidean 3-dim space are are "special relativities", and there are as many "special relativities" as there are parametric maps; in fact, an uncountable infinity of them.

3) Distortions introduced by "special relativities" are common distortions of maps between not isometric spaces, in our case between Lobachevskian negatively curved space $K<0$ and Euclidean space $K=0$.

4) Since "special relativities" are not unique, it implies that any relativistic physics (High Energy Physics) based on a particular "special relativity" is not unique as well.

Now, we will discuss consequences for science and for physics in particular.

\section{Isomorphism between ESR and BSR "Special Relativities"}

To review, the coordinates in Lobachevskian velocity space that are used in this work are:

1) Projective (homogeneous) Weierstrass coordinates $u\left(u_{0}^{2}, u_{1}^{2}, u_{2}^{2}, u_{3}^{2}\right)$, in the form $u_{0}=\frac{1}{\sqrt{1-\mathrm{v}^{2}}}, \quad u_{i}=\frac{\mathrm{v}_{i}}{\sqrt{1-\mathrm{v}^{2}}},|\mathrm{v}|<1, i=1,2,3$, normalized $u^{2}=u_{0}^{2}-u_{1}^{2}-u_{2}^{2}-u_{3}^{2}=1$, as per Formula (55).

2) Projective (homogeneous) rational coordinates used by authors to parametrize unit hyperboloid $u^{2}=u_{0}^{2}-u_{1}^{2}-u_{2}^{2}-u_{3}^{2}=1, u_{0}=\frac{1+v^{2}}{1-v^{2}}, u_{i}=\frac{2 v}{1-v^{2}}$, $|v|<1, \quad i=1,2,3$, normalized $u^{2}=1$.

For instance, in an abstract Lobachevskian plane $L^{2}$ creates images in the Euclidean plane $E^{2}$ by specifying coordinates in $E^{2}$ within the range of ad- 
missible values, see e.g. [16]. Thus the coordinates in an open disc representation (without a boundary at infinity) of a Lobachevskian plane are:

1) Coordinates in the disc $D^{2}$ of the form $\mathrm{v}_{x}=\tanh \theta \cos \alpha$, $\mathrm{v}_{y}=\tanh \theta \sin \alpha, \quad \mathrm{v}_{x}^{2}+\mathrm{v}_{y}^{2}<1,0 \leq \alpha \leq 2 \pi$ result in the Beltrami-Klein model of the Lobachevskian velocity plane.

2) Coordinates in the disc $D^{2}$ of the form $v_{x}=\tanh \frac{\theta}{2} \cos \alpha$, $v_{y}=\tanh \frac{\theta}{2} \sin \alpha, v_{x}^{2}+v_{y}^{2}<1,0 \leq \alpha \leq 2 \pi$ result in the Poincare model of the Lobachevskian velocity plane.

The angular coordinate $\alpha$ is irrelevant to our discussion and will be omitted.

As we have seen, different models (maps) of Lobachevskian geometry in Euclidean space show different distortions. All effects of "special relativities", either ESR or BSR, are just distortions caused by the mapping of a Lobachevskian, negatively curved (velocity) space into a flat space. We already noted that in ESR velocity $\mathbf{v}$ and intrinsic Lobachevskian velocity $\theta$ are related as $\mathbf{v}=\tanh \theta$, while in our BSR this relation is $v=\tanh \frac{\theta}{2}$.

Since:

$$
\text { Beltrami-Klein } \ni \mathbf{v}=\tanh \theta=\frac{2 \tanh \frac{\theta}{2}}{1+\tanh ^{2} \frac{\theta}{2}}=\frac{2 v}{1+v^{2}}, v \in \text { Poincare }
$$

and since (inverse map)

$$
v=\tanh \frac{\theta}{2}=\frac{\tanh \theta}{1+\sqrt{1-\tanh ^{2} \theta}}=\frac{\mathbf{v}}{1+\sqrt{1-\mathbf{v}^{2}}}
$$

we see that the velocity $\mathbf{v}$ measured in experiments due to ESR and the velocity $v$ measured in experiments due to BSR are mutually related by (88) and (89), which are known as isomorphism maps between the Beltrami-Klein ball model (v) of Lobachevskian geometry and the Poincare ball model of Lobachevskian geometry. It follows that all formulas of Einstein's ESR regarding velocities and their functions (or metric relations and their functions) due to the Beltrami-Klein model can be converted to formulas in our BSR due to the Poincare model (and vice-versa) via respective substitutions (88) and (89).

Proposition 20 The Poincare model of Lobachevskian geometry corresponds to the double sided action of the Lorentz group on Lobachevskian space while Beltrami-Klein model of Lobachevskian geometry corresponds to the left action of the Lorentz group on Lobachevskian space. The isomorphism between these two actions is realized by $\tanh \theta \Leftrightarrow \tanh \frac{\theta}{2}, \theta \in[0, \infty)$ given explicitly by the correspondence Formula (90) below:

$$
\left(\begin{array}{cc}
\frac{1+v^{2}}{1-v^{2}} & \frac{2 v}{1-v^{2}} \\
\frac{2 v}{1-v^{2}} & \frac{1+v^{2}}{1-v^{2}}
\end{array}\right) \Leftrightarrow\left(\begin{array}{cc}
\frac{1}{\sqrt{1-\mathrm{v}^{2}}} & \frac{\mathrm{v}}{\sqrt{1-\mathrm{v}^{2}}} \\
\frac{\mathrm{v}}{\sqrt{1-\mathrm{v}^{2}}} & \frac{1}{\sqrt{1-\mathrm{v}^{2}}}
\end{array}\right)
$$


The LHS of (90) is the matrix from the "special relativity" of Lobachevski Poincare - von Brzeski (77), $\left(t^{\prime}, x^{\prime}\right) \rightarrow(t, x)$, based on the present work. The RHS of (90) is the matrix from the "special relativity" of Einstein (Lobachevski Beltrami-Klein). The double sided arrow in the middle of (90) shows the isomorphism of the mappings, and can be shown as follows:

1) Mapping from Einstein to von Brzeski, or ESR $\Rightarrow$ BSR.

Take Equation (42) or (88) of the isomorphism $\mathrm{v}=\frac{2 v}{1+v^{2}}$ relating the Beltrami-Klein and Poincare models of Lobachevskian geometry and substitute it into all entries in the RHS of (92), i.e. into ESR. Simple calculations will result in the LHS (von Brzeski) matrix, i.e. BSR.

For example:

$$
\frac{1}{\sqrt{1-v^{2}}}=\frac{1}{\sqrt{1-\frac{4 v^{2}}{\left(1+v^{2}\right)^{2}}}}=\frac{1}{\sqrt{\left(\frac{1-v^{2}}{1+v^{2}}\right)^{2}}}=\frac{1+v^{2}}{1-v^{2}}
$$

and:

$$
\frac{\mathrm{v}}{\sqrt{1-\mathrm{v}^{2}}}=\frac{1+v^{2}}{1-v^{2}} \frac{2 v}{1+v^{2}}=\frac{2 v}{1-v^{2}}
$$

2) Mapping from von Brzeski to Einstein, or BSR $\Rightarrow$ ESR:

Take Equation (89) of the isomorphism $v=\frac{\mathrm{v}}{1+\sqrt{1-v^{2}}}$ between the Poincare and Beltrami - Klein models of Lobachevskian geometry and substitute it into all entries in the LHS of (90), i.e. into BSR. This will convert it into the RHS of (90), i.e. ESR. We leave the calculation to the reader.

It is quite remarkable that in the course of the present work on physics, the isomorphism Formulas (88) and (89), which are in the domain of pure geometry, were "rediscovered" by means of physics. It shows the deep and amazing interconnection between the abstract world of (Lobachevskian) geometry and the material world of physics.

\section{Homotopy of Maps. Uncountable Infinity of "Special Relativities". Undecidability of High Energy Physics}

First, if we are interested in High Energy Physics (HEP), we have to say what kind of physics this is exactly. To do so, we will first define "low energy physics".

Definition 21 Low Energy ("Non Relativistic") Physics is physics modeled on Euclidean geometry.

Low energy physics deals in small distances in Lobachevskian velocity/ momentum space $S L(2 C) / S U(2)$. Next, we need to explain what we mean by "large distances" and "small distances". In Euclidean space adjectives such as "large", "small" are meaningless. They carry zero information associated with them. However, in Lobachevskian spaces they have a very definite meaning. The key is the value of the negative curvature. Note that the Gaussian curvature of 
Lobachevskian velocity space is $K=-c^{-2}$. It follows that low distances will be those distances represented by relative velocities much less in comparison with $c$, or equivalently when the curvature $K$ is close to zero. In other words, when viewed locally, the curvature of Lobachevskian space may be disregarded. Physics in such domains may be regarded as Euclidean, i.e. "low energy", physics.

Definition 22 High Energy (“Relativistic") Physics is physics modeled on Lobachevskian geometry.

This is because energy is determined by distance in Lobachevskian velocity/momentum space $S L(2 C) / S U(2)$. The larger the distance, the larger the energy associated with it. Thus HEP is the physics of large distances in $S L(2 C) / S U(2)$. Accordingly, high energy physics is physics when the negative curvature of Lobachevskian space $S L(2 C) / S U(2)$, in either coordinate representation or in velocity/momentum representation, must be taken into account.

We already introduced the notion of a homotopy of maps in Section 2.3 in an informal way. In this section, homotopy will be used to prove the existence of an uncountable infinity (continuum) of "special relativities" and consequently an uncountable infinity of high energy relativistic) physics. We begin with the definition of homotopy.

Definition 23 Two continuous mappings $f$ and $g$ are homotopic (form a homotopy) if there exists a continuous mapping $h_{t} \times[0,1]$ such that $h(t=0)=f$ and $h(t=1)=g$.

The mapping $h$ as per definition 23 is represented by the Equation (93) below.

$$
h(t)=(1-t) f+\operatorname{tg}, t \in[0,1]
$$

Since the unit segment $[0,1]$ of the real line contains an uncountable infinity (continuum) of points, it follows that there is an uncountable infinity of maps which are in one-to-one correspondence with points $t \in[0,1]$. Maps related by a homotopy are called homotopy equivalent, in a similar way as topological spaces are equivalent when related by a homeomorphism. Equivalence in this context is understood in its standard mathematical sense. Equation (93) has simple intuitive meaning. Starting with the map $f$ at some real parameter $t=0$ we continuously deform the map $f$ until it becomes the map $g$ at some other value of a real parameter $t=1$.

With respect to "special relativities" BSR and ESR given by stereographic and gnomonic maps respectively, and represented by Poincare and Beltrami-Klein models of Lobachevskian geometry, respectively, we have the following theorem. Recall that the Poincare model is conformal while the Beltrami-Klein is not.

Theorem 24 There exists an uncountable infinity of non-isometric and non-conformal models of Lobachevskian geometry and the uncountable infinity (continuum) of "special relativities" built upon them. Any such hybrid (mixed) model is in one-to-one correspondence with the some point $t \in[0,1]$ in Equation (93).

Proof. Directly from definition of homotopy. 


$$
h(t)=(1-t) \tilde{u}^{*} \tilde{x}^{\prime} \tilde{u}+t \tilde{u} \tilde{x}^{\prime}, \quad t \in[0,1]
$$

We see that the above mapping (94) obeys the definition of homotopy. At $t=0, h(0)$ we have a stereographic map $\tilde{u}^{*} \tilde{x}^{\prime} \tilde{u}$, while at $t=1, h(1)$ we have a gnomonic map $\tilde{u} \tilde{x}^{\prime}$, and there is an uncountable infinity of mixed (hybrid) maps, neither stereographic nor gnomonic, in between. In this way, the Poincare model is continuously deformed into the Beltrami-Klein model, or in terms of special relativities, our "relativity" BSR is continuously deformed into Einstein's "relativity" ESR.

The message from theorem 24 is very disturbing. If there were only two options, we could run an experiment to see which option might better fit a particular condition. If the number of options would be (arbitrarily) finite, we could "in principle" verify which map best fit the experiment. But if the number of options is uncountably infinite, we unfortunately cannot run an infinity of experiments even in principle since it obviously would require infinite time. Thus experiments in HEP show only one of infinitely many faces of reality, and one point of infinitely many possibilities cannot be regarded as the ultimate truth.

Conclusion 25 (Incompleteness of HEP) Based on assumptions. $x^{2}=1=$ invariant,$c^{2}=1=$ invariant , under the isometries of 3-dim real Lobachevskian spaces $L_{X}^{3}$ and $L_{V}^{3}$ executed by the Lorentz group $S L(2 C)$, the knowledge acquired from "relativistic" or high energy physics is inconclusive and/or incomplete.

To see the validity of the above conclusion, consider a typical experiment in "relativistic" particle physics: the decay of unstable particles. Let take the well known decay of $\pi^{0}$ into two photons, $\pi^{0} \rightarrow 2 \gamma$. The experiment, in an Euclidean laboratory frame, results in some lifetime data, i.e. some real number $t_{\pi^{0}}$. Now we need to calculate back (interpret): what is the lifetime of $\pi^{0}$ in its frame, i.e. in the momentum hyperboloid which is Lobachevskian space. If the calculations are done in Beltrami-Klein model (or ESR), we obtain some value, say $t_{B K}$. If the calculations are done using the Poincare model (BSR), we will obtain a different value $t_{P} \neq t_{B K}$. So what we end up with is a $\pi^{0}$ meson with two different lifetimes which is obviously impossible. Furthermore, the same argument applies to momenta, energy, polarizations, reaction cross-sections, angular distribution of reaction products (since ESR is non-conformal while BSR is conformal), and so on. As we noted above, the situation is even more vague since there is in fact an infinity of mixed (hybrid) "relativities".

Remark 26 (Analogy with Quantum Mechanics) It is well known that quantum mechanics deals with states which can be either pure states or mixed states. If we label, for instance the Beltrami-Klein model as a pure state $\Phi_{B K}$ and Poincare model as a pure state $\Psi_{P}$, then the mixed state, or hybrid model mentioned here will be, $F=p_{1} \Phi_{B K}+\left(1-p_{1}\right) \Psi_{P}$, where $p_{1}$ and $1-p_{1}=p_{2}$ are real, non-negative numbers $0 \leq p_{1}, p_{2} \leq 1$ representing probability amplitudes of finding the mixed state in one of the pure states $\Phi_{B K}$ or $\Psi_{P}$. It is 
clear that there exists a continuum of such hybrid models or mixed states. For example, if $p_{1}=\frac{1}{2}$ and $p_{2}=1-\frac{1}{2}=\frac{1}{2}$ then we have a mixed state representing the hybrid model, which is neither a Poincare model nor a Beltrami-Klein model of Lobachevskian geometry. It follows that maps viewed as a physical states, which are homotopy related or which are of the same homotopy type, will produce isomorphic physics. We know that in quantum mechanics mixed states are represented by density matrices. Hybrid "relativities" can be represented in the same way which makes "classical physics" as probabilistic as quantum physics. This is an extremely interesting insight into "quantum classical worlds", which dismisses the common belief that quantum physics is probabilistic while classical physics is deterministic.

\section{Paradoxes as Distortions of Maps between Non-Isometric Spaces}

When humans explore the world around them, they encounter new phenomena, and use mathematical tools, e.g. maps, to make sense of the new phenomena. But if incorrect maps are used for such exploratory experiments, bizarre conclusions will result. Unfortunately new phenomena are often judged and interpreted in terms of already existing knowledge, which is frequently incompatible with experimental results. Sometimes, as was the case with quantum mechanics, a new fruitful approach emerges, but in many cases, the incompatibility between existing science and new theoretical or experimental facts leads to bizarre and false conclusions.

In this section, we prove the apparent nature of the so called Twin Paradox, and we resolve (in general) the problem of the shape of a circular fast moving object.

\subsection{The "Twin Paradox", Its Origin, and Its Trivial Solution}

The Twin Paradox is as old as ESR itself and so far there is no satisfactory and reasonable solution of the problem. It is interesting that Einstein himself never gave any solution to Twin paradox in the "special relativity" he authored. The solution presented here is the first and only one in the literature on the subject which is mathematically sound and does not involve any subjective factors.

We start with the standard scenario, but since we have already introduced the isomorphism between ESR and BSR, we proceed with a symmetrical treatment of both twins A and B, referenced to the same stationary clock C. Doing so removes all kinds of arguments based on asymmetry, e.g. traveling versus non-traveling twin, still present in literature.

At a fixed time, say "time zero" $t_{0}$ on an Earth clock C, both twin A and twin $B$ start their journey in the same fast rocket and are subjected precisely to the same conditions. Upon returning to Earth, and before seeing clock C, the twins use isomorphic relativities and calculate what each will see on clock C. Note that 
all three objects, twin $\mathrm{A}$, twin $\mathrm{B}$, and clock $\mathrm{C}$ are in the same place and are mutually motionless.

Twin A makes his calculation due to the Lobachevski-Beltrami-Klein model (ESR), and expects that the clock will show the time as $t_{0}+\Delta t_{E S R}$. Twin $\mathrm{B}$, who knows of Poincare model, makes his calculations of temporal distortions due to Lobachevski-Poincare-von Brzeski model (BSR), and expects that the clock will show the time as $t_{0}+\Delta t_{B S R}$. Since $\Delta t_{E S R} \neq \Delta_{B S R}$ it implies that

$t_{0}+t_{E S R} \neq t_{0}+\Delta_{B S R}$, which in turn implies that the clock should display two different readings - an impossible outcome, i.e. a "paradox". Obviously, the clock will display a single reading at the same instance when twins A and B look at it. The state of the clock, i.e. the real number on the clock's display, does not depend on an identical copy of an observer (twin) who is looking at it. Needless to say, this will cause much confusion for both twins.

Next, when twin A and twin B attempt to calculate their ages due to different isomorphic "relativities" (maps they used in their journeys), they will discover that their ages are no longer the same, despite the fact that they underwent precisely the same journey and were subjected to precisely to the same conditions.

Using Formulas (58) and (84), we can summarize the situation as follows:

1) If $\Delta t_{A}^{\prime}=\Delta t_{B}^{\prime}$ it implies that $\Delta t_{A} \neq \Delta t_{B}$,

2) If $\Delta t_{A}=\Delta t_{B}$ it implies that $\Delta t_{A}^{\prime} \neq \Delta t_{B}^{\prime}$.

In other words, after the trip(s), we will obtain quite bizarre results: either the clock will show double readings or twins $A$ and $B$ have two different ages. This is obviously an impossible outcome showing the apparent nature of distortions resulting from non-isometric maps called "special relativities".

The so called Twin Paradox is not real and it is due to apparent distortions only. The paradox is apparent in the same way as the shape and size of Greenland is different on two (homotopic) maps; see Figure 1. Distortions introduced by various maps of curved space into a flat space are of apparent character only and should not be viewed as something real.

The following example is even more evident in its outcome. It is in essence what high energy particle physicists confront routinely in their work (we will return to this when we discuss problem with lifetimes of unstable particles.

Since we have alternative "relativities", we do not need twins, and we limit our consideration to one traveler only. A traveler takes a trip in a rocket. After the trip (returning to Earth), the traveler is asked about his or her age. The traveler has many options for the answer. One option is to calculate the age due to ESR (gnomonic map); another option is to calculate the age due to BSR (stereographic map); furthermore, the traveler has an infinity of options to calculate the age due to the hybrid maps in between. As a result, the traveler is unable to give a definite answer. Then, the traveler is asked again: who might know your age? The answer is, that after the trip nobody really knows the actual age of the traveler. Problems of this sort are called undecidable problems. A theory which contains undecidable problems is incomplete. 
Remark 27 A reader familiar with quantum mechanics is familiar with the dilemma known as "Schrodinger's cat" regarding the incompleteness of information due to a quantum mixed state. If we replace the Earth clock $C$ in the above analysis with the cat (biological clock), we will come to the analogous situation of inconclusiveness of the state of the cat prior to actually seeing it. One of the twins will claim that the cat is already dead while the other will claim that the cat is still alive. Since both claims are due to isomorphic "relativities" of equal logical value, we arrive at a state of the cat which is half dead and half alive. The only possibility is to observe the cat after returning from the trip (collapse of the wave function by breaking the cat box) and checking the state of the cat.

Conclusion 28 Relativistic physics contains undecidable problems, and therefore is an incomplete theory.

The close resemblance here with quantum mechanics (QM) is striking. The information about the traveler's age is lost due to the geometry of mixed maps (mixed states in QM). This clearly shows that what we have here is the quantum mechanical - probabilistic case rather than "classical" deterministic case. It is ironic that Einstein who was hostile of quantum mechanics developed his ESR not realizing that ESR is just a "pure state" (or "pure map"), one of infinitely many, in a more general scheme which is inherently probabilistic and in which the outcome of a physical process is specified not by a single real number but by probability amplitudes for alternative outcomes.

\section{2. "Wheels of a Fast Moving Bicycle" and Undecidable Questions in High Energy Physics}

Regarding the shape of fast moving circular objects ("bicycle wheels") it is easy to see that all definite conclusions up to now, in one way or the other, are equally wrong. This means that all authors who conclude that a fast moving circular object will appear as circular, or those who conclude they will appear as not circular (e.g. elliptical), are equally wrong. This is because the question about the shape of fast moving spherical/circular object is undecidable, which means that it is impossible a-priori to give a definitive answer to this question. Undecidability here is of the same sort as the undecidability about the traveler's age we discussed in Section 7.1, however, it follows from the conformality or non-conformality properties of different maps.

Conformal maps are maps which preserve angular relations, so shapes of objects in images remain unchanged however the size of the objects change. Non-conformal maps do not preserve angular relations and consequently shapes of objects in images will be deformed. It is well known that the stereographic map is conformal while the gnomonic is not. The Poincare model of Lobachevskian geometry, and BSR which is a result of a stereographic projection are conformal, while the Beltrami-Klein model of Lobachevskian geometry and ESR which results from a gnomonic projection are non-conformal. Therefore, there is no way to conclude a-priori anything definite about the shape of a fast moving circular object because the definite "truth" depends on which map of 
Lobachevskian velocity space or which model of "relativity" is used.

Suppose you have two cameras, one producing Poincare images or maps, and the other one producing Beltrami-Klein images or maps onto a piece of flat paper. ("Camera" here does not refer to a piece of hardware that takes pictures, but as a mapping or algorithm which maps objects between spaces of different curvatures). If you use the Beltrami-Klein camera, the images of a fast moving circle will be ellipses and not circles. This is because Beltrami-Klein model of Lobachevskian geometry (used in ESR) is a non-conformal model. On the other hand, if you use the Poincare camera, the images of fast moving circles will be circles, not ellipses. This is because Poincare model is conformal. Lobachevskian circles (spheres) in the Poincare model are also the Euclidean circles (spheres); there is distortion in size but not in a shape, a fact well known in non-Euclidean geometry. We have the following theorem:

Theorem 29 It is impossible a-priori to determine the shape of fast moving circular (spherical) objects. The shape of fast moving circular objects is model dependent. In a Lobachevski-Beltrami-Klein-Einstein map, the image of a fast moving circle will be an ellipse. This is because the Beltrami-Klein model used in ESR is a non-conformal model. In the Lobachevski-Poincare-von Brzeski map, the image of a fast moving circle will be a circle. This is because the Poincare model is a conformal model.

Proof. Directly from properties of non-conformality/conformality of Beltrami-Klein and Poincare models (Figure 8 and Figure 9).

Conclusion 30 The inability to answer the question regarding the shape of a fast moving circular object is a result of incompleteness of information. In fact we proved that questions about the world of high relative velocities, or questions about the world of high energy physics, are a-priori undecidable. Answers to those questions are decidable only up to a homotopy of maps.

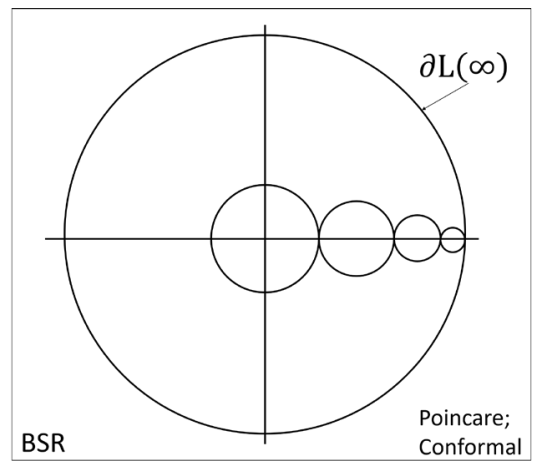

Figure 8. The apparent shape of "fast moving" spherical (circular) objects due to Poincare model of Lobachevskian geometry and the associated with it version of our "special relativity" - BSR. Since the model is conformal, spheres (circles) will appear in preserved shapes but in decreased sizes as distance (relative velocity) in Lobachevskian velocity/momentum space increases. The apparent size of objects close to the boundary at infinity, which is the sphere $S^{2}$ (circle $S^{1}$ in the above figure), will be arbitrarily small point-like. The same reasoning applies to Lobachevskian position space - Lobachevskian Universe, and can be viewed as a real example of Lobachevskian cartography. 


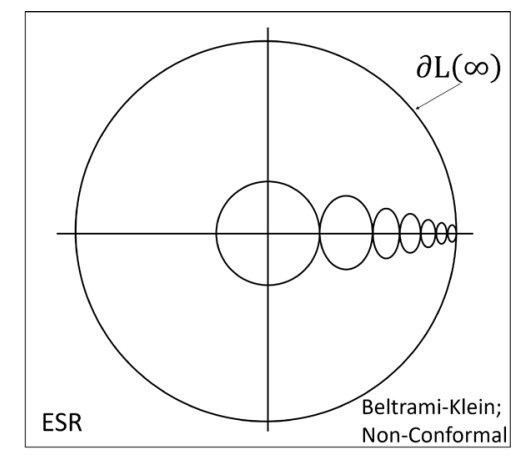

Figure 9. The apparent shape of "fast moving" spherical (circular) objects due to Beltrami-Klein model of Lobachevskian geometry and the associated Einstein's "special relativity" - ESR. Since this model is non-conformal, as relative velocity (with respect to the center) increases, spheres (circles) will appear: a) flattened (ellipsoidal) and b) smaller in size. The apparent shape and size of objects close to the boundary at infinity (circle $S^{1}$ in the above figure) will be very "thin" and very "small", respectively. The same argument holds for a Lobachevskian Universe if perceived via the Beltrami-Klein model, and can be viewed as a real example of Lobachevskian cartography.

\section{Application to Astrophysics}

Deep space astrophysics shows that geometry of large scale vacuum (background geometry) of the Universe is Lobachevskian [4] [5] [6] [7]. Therefore we can ask a question. How do we perceive the Lobachevskian Universe around us, via the Beltrami-Klein model or via the Poincare model, or via a mixed model? This is an interesting question which can be resolved experimentally.

As we already mentioned, Lobachevskian geometry in physics is represented by velocity space $L_{U}$ and by position (coordinate) space $L_{X}$ which we identify with the large scale vacuum - Lobachevskian Universe. We know that galaxies in the Universe are basically of two shapes, spherical and elliptical. If the Universe appears to us as in the Poincare model, then statistical distribution of galactic shapes will not depend on distance. Independently of how far we can see, there will on average be the same count of spherical and elliptical galaxies. That is because Poincare model is conformal, spheres are mapped onto spheres, ellipsoids are mapped onto ellipsoids.

On the other hand if space appears to us as the Beltrami-Klein model, the distant spherical galaxies will look more and more elliptical. So the number of elliptical galaxies will rise with distance. However things are more complicated since depending on galaxy orientation with respect to an observer, some elliptical galaxies will look even more elliptical while others will appear less elliptical, or maybe even as spheres.

Moreover, there is still an infinity of mixed models which makes things even more complicated. The problem is not easy to resolve but we hope that astrophysicists with help of advanced statistical analysis will be able to solve it.

\subsection{The Meaning of Distortions for Physics. What Is Real and What Is Apparent?}

Everybody has their own sense of reality and we will not comment on that. 
However, the adjective "apparent" has more of an ambiguous meaning for each person. Therefore we take a definition of "apparent" from Webster's New Collegiate Dictionary.

Apparent (illusory, seeming, ostensible): manifest to the senses or mind as real or true on the basis of evidence that may or may not be factually valid.

The main source of confusion about what is real and what is apparent is that we are comparing metric relations (sizes) in non-isometric spaces, e.g. comparing "apples and oranges". We cannot reach conclusions about sizes between spaces of different curvature which are not isometric. While we can go directly to Greenland to verify our data regarding cartography from spheres, we cannot go to a Lobachevskian negatively curved space and to make direct measurements there. What we can account for at most is that we have to work with distorted images in our 3-dim Euclidean world. Maps (projections) between curved and flat spaces result in distortions and there is no way to circumvent this misfortune. The reader can now appreciate why we devoted a considerable portion of this paper to maps (projections) from curved to flat spaces.

The fundamental misunderstanding about Lorentz transformations which plagues all of physics since 1905 is that they introduce distortions. This is false. Lorentz transformations are isometries of Lobachevskian space and hence are distortion-less. Distortions are introduced when we map (project) information from a hyperboloid into our Euclidean laboratory. "Paradoxes" result from incorrect interpretation of "special relativities" via flat Minkowski pseudo-Euclidean geometry. In the interpretation of "special relativities" as maps from Lobachevskian into Euclidean space no "paradoxes" are present.

We've come to the point where we have to explain which effects of "special relativities" can be regarded as real, and which ones are only apparent. Recall that since it's publication in 1905 , some phenomena related to "special relativity" have been viewed as paradoxical. Moreover, due to formulation of "special relativity" via a Minkowski flat space, the origin and nature of such paradoxes were entire obscure. Our exposition makes it easy to understand the origin of such paradoxical phenomena, and below we give our view on what is real and what is not when dealing with "special relativities".

If we admit that the velocity of light in a vacuum is a limiting value, i.e. $c=1$, and we admit the independence of the velocity of light (in a vacuum) with the state of motion, $c \pm u=c$, then space of velocities has a natural mathematical model as Lobachevskian (hyperbolic) 3 dimensional space, and its group of isometries is the Lorentz group $S L(2 C)$. From here, due to various Lorentz group actions on Lobachevskian, space we obtain various "special relativities", each identified as some map from Lobachevskian $K<0$ into Euclidean space $K=0$. Thus it is natural to discuss the distortions or deformations such maps introduce. Since each map is between a pair of spaces of different curvature which are not isometric, the situation with distortions is more subtle than it might initially appear. 
In fact two kinds of distortions of different origin have to be considered:

1) Since Lobachevskian space is more volumetric than Euclidean space (see Table 1), images from Lobachevskian space recorded in Euclidean space, by necessity, will be compressed. It should be noted that the effect of compression is regarded as the compression of images, and not as a compression of material bodies. This effect is independent of the map used, and is a consequence of pure geometry. For this reason we think that effects of image compression might be regarded as real since they are given by Nature itself and they cannot be eliminated by man in any way. On the other hand, it has to be clearly understood that a comparison of metric relations (sizes) in two non-isometric spaces having different volumetric content and having two different units of length, is highly misleading.

2) In addition to distortions (deformations) of images resulting from different curvatures, there are "technical" (man-made) distortions resulting from the particular type of map employed in the projection of images from curved into Euclidean space. It has been shown that for instance gnomonic and stereographic maps introduce different distortions when mapping the same curved space into a flat space; see Figure 1(a) and Figure 1(b). The distortions resulting from the type of a map are purely apparent, i.e. not real, and may be altered by choosing an appropriate (to an experiment) map.

As a result, in any experiment at high energies (large distances in Lobachevskian space), we record a mixture of such real and apparent distortions, which cannot be untangled-the only option we have in order to obtain information from a negatively curved space is to employ some kind of map! Thus, images (data) which we record in "relativistic physics" should be regarded as "real/apparent" images (data). To which degree the images are real or cannot be separated in a unique way.

\section{Summary}

In this paper, we presented the discovery of the non-uniqueness of Einstein's "special relativity" and inconclusiveness of the High Energy Physics ("relativistic physics") resulting from different maps from Lobachevskian into Euclidean space. Different, yet homotopy equivalent, maps result in mathematically equivalent (isomorphic) but physically not-equivalent "special relativities" in that they will produce different numerical predictions. Another new result is the identification of "special relativities" as common maps from Lobachevskian spaces into Euclidean space, and their association with well known models of Lobachevsian geometry.

Einstein's "special relativity" (ESR) is not a theory. It is a gnomonic map from Lobachevskian space into Euclidean space, is one of infinitely many possible maps, and as such is not unique. From a mathematical point of view, it is based on the Beltrami-Klein model of Lobachevskian geometry. This Lobachevski-Beltrami-Klein-Einstein "relativity" is isomorphic, but it is not isome- 
tric and not conformal with the authors' BSR, what we call the LobachevskiPoincare-von Brzeski "relativity”. From the point of view of physics, Einstein's "special relativity" map corresponds to single sided motion. In physical applications, it is a natural choice for fixed target scattering experiments like those on accelerators with a fixed target.

The "special relativity" presented in this paper, namely Lobachevski-Poincarevon Brzeski (BSR) is not a theory either. It is a stereographic map from Lobachevskian space into Euclidean space and as such is also not unique. From mathematical point of view, it is based on a Poincare model of Lobachevskian geometry, which is isomorphic but not isometric with the ESR "relativity". From the point of view of physics, it describes two sided motion. It corresponds to center of momentum frame scattering, in accelerators with counter rotating beams. In astrophysics, it applies to so called "relativistic beams" ejected from galactic nuclei.

Between ESR and BSR, there is a continuum of hybrid "relativities" due to homotopy between maps, as was explained in the Section 6. Therefore, results acquired in High Energy "Relativistic" Physics are not conclusive. More precisely, they are conclusive up to homotopy only.

The results of experiments in High Energy Physics and deep space astrophysics depend on maps called "special relativities", which translate non-Euclidean reality into Euclidean data in our laboratory, data which are inevitably deformed due to different distortions introduced by those different maps.

Our "special relativity" BSR is equally valid from the logical, the mathematical, and the physical point of view. There are no criteria of any kind to discriminate BSR versus ESR since there are no criteria (beyond the matter of convenience in a particular situation) to discriminate the Poincare model versus Beltrami-Klein model of Lobachevskian geometry.

To our knowledge, the BSR alternative to ESR is presented here for the first time in scientific literature. "Alternative" has to be understood in the same sense as alternative maps are used in the practice of mapping the Earth's surface. As we said in the introduction, map making is mathematics. Navigation and exploration of the physical world, using those maps, is experimental physics.

Unfortunately, we cannot experience (perceive) global Lobachevskian geometry directly, but only via our local Euclidean reality. Our Euclidean experience is only projections - images or maps due to the Poincare model or due to the Beltrami-Klein model, or due to an infinity of homotopy equivalent hybrid models. Different maps or projections or images will result in different data from the world of high energy physics, and we do not have any "solid reference" to know their true nature (pre-images). We have no direct access to the Lobachevskian geometry "source code" of Nature and we must work with the Lobachevskian world in terms of its Euclidean images. The situation is reminiscent of Plato's Allegory of the Cave. The bird-eye's view of the presented paper is summarized in Table 2. 
Table 2. Bird's eye view of Einstein's and the authors' "relativities".

\begin{tabular}{ccc}
\hline & $\begin{array}{c}\text { Einstein } \\
\text { "Special Relativity" (ESR) }\end{array}$ & $\begin{array}{c}\text { von Brzeski } \\
\text { "Special Relativity" (BSR) }\end{array}$ \\
\hline Lobachevskian & $S L(2 C) / S U(2)$ & $S L(2 C) / S U(2)$ \\
Real 3-dim Space & Quotient of Lorentz Group & Quotient of Lorentz Group \\
Type of Lorentz Group & Single sided (left) & Double sided \\
Action on $S L(2 C) / S U(2)$ & $x=g x^{\prime}$ & $g^{*} x^{\prime} g$ \\
Type of Projection & Gnomonic (central), & Stereographic, \\
(Type of Map) & non-conformal & Projective rational \\
Type of Coordinates & Projective Weierstrass & Poincare, conformal \\
Resulting Model of & Beltrami-Klein, & non-conformal \\
Lobachevskian Geometry & Isomorphic as models, & Isomorphic as models, \\
Relation between BSR and ESR & Homotopic as maps & Homotopic as maps \\
\hline
\end{tabular}

\section{Conflicts of Interest}

The authors declare no conflicts of interest regarding the publication of this paper.

\section{References}

[1] Anderson, W.A. (1999) Hyperbolic Geometry. Springer, London, Berlin, Heidelberg. https://doi.org/10.1007/978-1-4471-3987-4

[2] von Brzeski, G. and von Brzeski, V. (2018) Journal of Modern Physics, 9, 13261359. https://doi.org/10.4236/jmp.2018.96081

[3] von Brzeski, G. (2008) Acta Physica Polonica, B, 39, 1501-1520.

[4] von Brzeski, G. (2007) Russian Journal of Mathematical Physics, 14, 366-369. https://doi.org/10.1134/S1061920807030107

[5] Borisovich, Yu., Bliznyakov, N., Izrailevich, Y.A. and Fomenko, T. (1985) Introduction to Topology (English edition). Mir Publishers, Moscow.

[6] Buseman, H. and Kelly, P.J. (1953) Projective Geometry and Projective Metrics. Academic Press, New York.

[7] Reynolds, W. (1993) The American Mathematical Monthly, 100, 442-455. https://doi.org/10.1080/00029890.1993.11990430

[8] Gelfand, I.M., Grayev, M.I. and Vilenkin, I.Y. (1966) Integral Geometry and Representations Theory. Translated from Russian, Academic Press, New York.

[9] Gelfand, I.M., Minlos, R.A. and Shapiro, Z.Y. (1963) Representations of the Rotation and Lorentz Groups and their Applications. Pergamon, New York.

[10] Gorbatsevich, V.V., Onischik, A.L. and Vinberg, E.B. (1997) Foundations of Lie Theory and Lie Transformations Groups. Springer Verlag, Providence, Rhode Island.

[11] Klein, F. (2000) Forlesungen Uber Nicht Euklidische Geometries. American Mathematical Society, New York, Berlin, Heidelberg.

[12] Greenberg, M. (1993) Euclidean and Non-Euclidean Geometries. 3rd. Edition, Development and History, W.H. Freeman and Company, New York.

[13] Einstein, A. (1905) Annalen der Physik, 322, 891-921. 
https://doi.org/10.1002/andp.19053221004

[14] Barrett, J.F. (1994) On Varicack's Interpretation of Special Relativity in Hyperbolic Space with Application to the Redshift. PIRT Conference, Imperial College, London, September 1994, 17-20.

[15] Varicak, V. (1912) On the Non Euclidean Interpretation of the Theory of Relativity. Translation from German. Jahresbericht der Deutschen Mathematiker Vereinigung 21.

[16] Ramsay, A. and Richtmayer, R.D. (1995) Introduction to Hyperbolic Geometry. Springer, New York, Berlin, Heidelberg. https://doi.org/10.1007/978-1-4757-5585-5 